\title{
GADIA: A Greedy Asynchronous Distributed Interference Avoidance Algorithm
}

\section{Citation}

Babadi, Behtash and Vahid Tarokh. 2010. GADIA: A greedy asynchronous distributed interference avoidance algorithm. IEEE Transactions on Information Theory 56(12): 6228-6252.

\section{Published Version}

doi:10.1109/TIT.2010.2081090

\section{Permanent link}

http://nrs.harvard.edu/urn-3:HUL.InstRepos:4687268

\section{Terms of Use}

This article was downloaded from Harvard University's DASH repository, and is made available under the terms and conditions applicable to Open Access Policy Articles, as set forth at http:// nrs.harvard.edu/urn-3:HUL.InstRepos:dash.current.terms-of-use\#OAP

\section{Share Your Story}

The Harvard community has made this article openly available.

Please share how this access benefits you. Submit a story.

Accessibility 


\title{
GADIA: A Greedy Asynchronous Distributed Interference Avoidance Algorithm
}

\author{
Behtash Babadi and Vahid Tarokh
}

\begin{abstract}
The problem of distributed dynamic frequency allocation is considered for a canonical communication network, which spans several networks such as cognitive radio networks and Digital Subscriber Lines (DSL). A Greedy Asynchronous Distributed Interference Avoidance (GADIA) algorithm for horizontal spectrum sharing has been proposed that achieves performance close to that of a centralized optimal algorithm. The convergence of the GADIA algorithm to a near-optimal frequency allocation strategy is proved and several asymptotic performance bounds have been established for various spatial configurations of the network nodes. Furthermore, the near-equilibrium dynamics of the GADIA algorithm has been studied using the Glauber dynamics, by identifying the problem with the anti-ferromagnetic inhomogeneous long-range Potts model. Using the near-equilibrium dynamics and methods from stochastic analysis, the robustness of the algorithm with respect to time variations in the activity of network nodes is studied. These analytic results along with simulation studies reveal that the performance is close to that of an optimum centralized frequency allocation algorithm. Further simulation studies confirm that our proposed algorithm outperforms the Iterative Water-filling algorithm in the low SIR regime, in terms of achieved sum-rate, complexity, convergence rate and robustness to time-varying node activities.
\end{abstract}

\section{Index Terms}

Dynamic spectrum allocation, interference avoidance, cognitive radios, distributed algorithms, Glauber dynamics.

Copyright (c) 2010 IEEE. Personal use of this material is permitted. However, permission to use this material for any other purposes must be obtained from the IEEE by sending a request to pubs-permissions@ieee.org.

B. Babadi and V. Tarokh are with the School of Engineering and Applied Sciences, Harvard University, Cambridge, MA, 02138. (e-mails: \{behtash, vahid\}@ seas.harvard.edu)

This research is supported in part by ARO MURI grant number W911NF-07-1-0376. The views expressed in this paper are those of the authors alone and not of the sponsor. 


\section{INTRODUCTION}

Dynamic frequency allocation has an important role in improving the performance of communication networks, for it results in less transmission power, which is a crucial objective in system design. To do this in an optimal way, there needs to be a centralized processor with full knowledge of the spatial distribution profile of the network nodes. However, in many emerging communication networks (such as ad hoc wireless networks, wireless sensor networks, cognitive radios, etc.), no central frequency allocation authority is naturally available. This makes distributed frequency allocation an important, but mostly unchartered territory in networking.

Centralized frequency allocation has been extensively studied in the context of cellular wireless systems (See, for example, [31] and [49]). As for wireless networks, Leung et al. [30] propose a heuristic centralized algorithm based on local search algorithms to search through the possible frequency combinations. In another approach, Steenstrup [48] introduces a central controller to the network, which assigns frequency bands to the nodes based on their interference with their neighboring high priority nodes.

There are also various proposed methods for decentralized (distributed) frequency allocation in different contexts (See, for example, [13], [15], [22], [28], [34], [37], [38], [40], [41], [42], [43], [45], [51], [56], [58], and [59]). These include methods based on graph coloring for cognitive networks, greedy interference avoidance techniques, Iterative Water-filling for Digital Subscriber Lines (DSL), game theoretic approaches to dynamics spectrum allocation and methods based on auction theory. Here, we review a number of these results which are most relevant to our work (For a more comprehensive review of these results, see [36]).

These approaches may either excessively simplify the interference models, or may not be decentralized, or may require too much information exchange between autonomous nodes/clusters, or may suffer from all these shortcomings. Additionally, they may be too complex to implement.

Ramanathan [43] takes an approach based on approximating the optimal resource allocation solutions on a graph. Peng et al. [38] propose that secondary users choose their spectrum according to their information about their local primary and secondary neighbors. They employ a simplified model for mutual interference of the network nodes that turns the problem into the graph multi-coloring problem. They subsequently compute a sub-optimal solution to the graph multi-coloring by using approximation algorithms to the graph labeling problem. Cao et al. [57] 
show that the graph model has the potential to represent the physical interference model. Zhao et al. [60] propose a distributed coordination protocol in order to construct an in-band control path (instead of pre-assigned out-of-band control channel) so that the nodes can coordinate and choose their spectrum accordingly. In a different approach by Cao et al. [12], the communication load of coordination between the nodes is decreased and a rule-regulated spectrum sharing mechanism is used, in which the nodes regulate their actions by complying with a set of predefined rules.

In another strand of work, Rose et al. [45], D. C. Popescu et al. [40], [41], and O. Popescu et al. [42] extensively study the problem of greedy interference avoidance in wireless networks. Rose et al. [45] propose and study iterative algorithms (namely, the eigen-algorithm and the MMSE algorithm) for interference avoidance through waveform shaping in a synchronous network of multiple users connected to a common receiver (or co-located set of receivers). D. C. Popescu et al. have extended the results of [45] to several scenarios in [40] and have been formalized them in the more general framework of multiple-access vector channels in [41]. It must be noted that the GADIA algorithm is an extension of the greedy interference avoidance procedure to the canonical network model considered in this paper.

In the context of Digital Subscriber Lines (DSL), some recent works regarding spectrum balancing have been done (See, for example, [13] and [58]). The objective of spectrum balancing in DSL systems is to maximize the throughput of each user by shaping its Power Spectral Density (PSD) of transmission, satisfying a certain power constraint. Yu et al. [58] propose the method of Iterative Water-filling in order to solve the problem. In the case of two users, they show the existence and conditions on the uniqueness of a Nash equilibrium point for the iterative algorithm. However, each user must know a weighted sum of the PSD of the other users (interference), in order to do water-filling. The Iterative Water-filling algorithm has high complexity and the resulting Nash equilibrium point is not necessarily the optimal solution. For instance, in a two-user scenario, if both users start with a flat PSD initially, iterative Water-filling does not change their PSD. This is clearly a Nash equilibrium point, but is far away from the optimal answer. Etkin et al. [15] and O. Popescu et al. [42] show that this non-optimal Nash equilibrium point might be the only Nash equilibrium, and therefore Iterative Water-filling fails for various scenarios.

Cendrillon et al. [13] consider the scenario where the users need to balance their power along a number of tones in order to optimize their throughout under power constraints. The optimization 
problem is relaxed by introducing a virtual user with fixed thresholds. It turns the problem into a separable optimization problem across the tones for different users. An algorithm has been proposed to solve the relaxed problem iteratively via solving local optimization problems by the users. The knowledge of a weighted sum of the PSD of the other users (as a measure of interference) is required for each user to solve its local optimization problem. The convergence of the algorithm has been shown in high SIR regime. Simulations show that the achievable region resulted by the solution of the relaxed distributed optimization is close to that of the optimal centralized solution. However, no one-to-one correspondence between the points of the achievable regions of the optimal (centralized) and decentralized algorithms is guaranteed. Therefore, the algorithm does not necessarily converge to optimal values. For the case of asynchronous transmission (in the presence of ICI), the optimization problem is not separable across the tones. They have therefore used heuristic optimization approaches with no convergence guarantees.

Etkin et al. [15] show that the problem of optimal PSD shaping across the users is reducible to that of allocating piece-wise constant powers. This result reduces the complexity of the spectrum sharing problem. Furthermore, a number of achievability and existence results in the context of non-cooperative and cooperative game theory for obtaining efficiency and fairness, as well as a punishment-based mechanism have been established. Another approach has been presented by Huang et al. [22], where each user in the network announces a price to the other users, so that they can adapt their power allocation accordingly. Convergence results have been established using supermodular game theory. Bae et al. [5] and Huang et al. [23] propose and investigate methods based on auction theory. In particular, Bae et al. [5] consider the scenario where a spectrum broker collects bids by the users and allocates the resource (power or bandwidth) according to a sequential second-price auction. Equilibrium analysis is presented for the two-user case and the existence of a Nash equilibrium has been established for the $n$-user scenario.

Hicks et al. [21], Menon et al. [33], Sung et al. [50], [51], and Ulukus et al. [53] consider spreading code adaptations, where each node is isolated in frequency and spreading codes are used to minimize the interference (See [36] for a detailed discussion of this topic). Lacatus et al. [28] present a distributed algorithm for codeword and power adaptation towards achieving a target SINR in CDMA systems, in the context of non-cooperative game theory. In [34], [35] and [37], methods based on potential games have been proposed. Nie et al. [37] propose a communication 
protocol in which the nodes can coordinate and obtain sufficient information for their decision making. Moreover, convergence to a mixed-strategy equilibrium has been established. Neel et al. [34] establish the convergence to a pure-strategy equilibrium, under the hypothesis of Bilateral Symmetric Interference (See Section III-A for more details).

In this paper, we consider a canonical network model which can be used to represent various communication networks. Examples are clustered wireless networks (where the agents are divided into different clusters and each cluster is represented by a cluster-head) and Digital Subscriber Lines. A Greedy Asynchronous Distributed Interference Avoidance (GADIA) algorithm has been proposed for the horizontal spectrum sharing in such networks. The GADIA algorithm provides a simple, fully distributed, dynamic frequency allocation strategy that requires neither any information exchange between autonomous devices, nor even any knowledge of the existence of other autonomous entities. Additionally, it can be used in conjunction with any realistic wireless radio channel model such as those commonly employed in wireless standards (Hata model, Okumura model, etc.).

In the GADIA algorithm, each node, having knowledge about the interference it experiences, chooses the frequency band with the least amount of interference from the other nodes. It is shown that the GADIA algorithm converges to a near-optimal spectrum assignment (under the hypothesis of interference reciprocity), without any cross-cluster information exchange. In [34] and [36], the convergence of a GADIA-like algorithm is established under the hypothesis of Bilateral Symmetric Interference (BSI). The network utility in [34], [35] and [36] is the negated sum of interferences in the network, which has also been employed by the present authors in [2], [3] and [4]. To guarantee convergence under BSI, the measured marginal interference contribution between pairs of radios (clusters) needs to be the same so that in [34] and [36], one needs to have $P_{i} \alpha_{i j} f\left(s_{i}, s_{j}\right)=P_{j} \alpha_{j i} f\left(s_{j}, s_{i}\right)$ for all $i$ and $j$, where $P_{i}$ is the transmittion power of node $i, \alpha_{i j}$ is the normalized channel between nodes $i$ and $j$, and $f(\cdot, \cdot)$ is the interference leakage function (See Section II for more details). In general, the BSI condition does not hold in a network with generic power distribution over the users. In [35], this is overcome by having each radio, $i$, scale its metric by its own transmit power $P_{i}$; [35] shows that this is sufficient to satisfy BSI with a generic power distribution. Thus [35] and GADIA differ only in that [35] scales the metric by $P_{i}$ while GADIA does not. In the nomenclature of [35], GADIA would be considered a weighted potential game while [35] is an exact potential game, with [35] having 
a potential function (called network utility function herein) given by Eq. (6) divided by 2. In practice, this distinction means that GADIA saves a multiplication for every channel interference evaluation, while converging to the same operating points whereas the linear-space properties of exact potential games allows [35] to aggregate interference measurements from each device in a cluster for more device-specific responsiveness. This work also establishes performance bounds on the GADIA algorithm (Section IV) and considers continuous time dynamical analysis (Section V) which were not considered in [34], [35] and [36], and to the best of our knowledge in any existing work on this topic. In particular, several asymptotic performance bounds for a wide range of network topologies have been established using the symmetries of the network utility and the minimum energy property of Bravais lattices. The network utility, under which the performance of the GADIA algorithm is studied, is the weighted aggregate interference. It is shown that this network utility is closely related to the sum-rate of the network. Furthermore, the dynamical behavior of the GADIA algorithm has been comprehensively studied, in analogy to the Glauber dynamics of the inhomogeneous long-range anti-ferromagnetic Potts model. In particular, we have constructed a framework based on stochastic analysis in order to evaluate the near equilibrium performance of the GADIA algorithm, in presence of time-varying activity of the network nodes. Simulation results (Section VI) show that the GADIA algorithm achieves more than $90 \%$ of the optimal sum-rate of the network in the low SIR regime, for various network topologies. Also, further simulation studies confirm the robustness of the GADIA algorithm with respect to the time-varying node activities, in accordance with the analytical results.

The main contributions of this paper are: (1) proposing a simple, low-complexity, robust and fully decentralized algorithm for horizontal spectrum sharing, (2) proving explicit performance bounds on the outcome of the algorithm in various environments, (3) presenting an analytical model for the dynamics of the proposed algorithm, inspired by methods from statistical physics, and (4) analytical evaluation of the robustness of the algorithm in presence of time-varying node activities.

The outline of this paper follows next. In Section II, the canonical network model, the network utility formulation and the underlying assumptions are discussed. The GADIA algorithm is introduced in Section III, followed by the discussion of its implications. The convergence of the GADIA algorithm and asymptotic performance bounds are established in Section IV. Section $\mathrm{V}$ includes the study of the dynamical properties of the GADIA algorithm, such as the near- 
equilibrium dynamics and performance evaluation under time-varying activity of network nodes. Simulation studies are presented in Section VI, followed by conclusion in Section VII. Finally, a number of technical lemmas and mathematical derivations are presented in Appendices A and B.

\section{CAnOnical Network Model}

Suppose that we have a set of network nodes distributed in space. In general, each node may have an internal structure, i.e., it may comprise smaller entities. Each node is interested in inter-node and/or intra-node communications. We denote this general structured network by the canonical network. Many communication networks of interest can be represented in this canonical form. As mentioned in the introduction, two such networks are the following:

Clustered Wireless Networks: Suppose that we have a set of transceivers distributed in space such that they can be partitioned into a union of possibly overlapping clusters. Each cluster is equipped with a cluster-head. These networks often happen in practice. For instance, in a combat scenario, a group of soldiers can be divided into a number of clusters according to their missions. We briefly review a few examples of such networks in what follows.

Moreover, such networks commonly arise in the context of cognitive radios. According to the recent FCC order on TV white spaces [16], all fixed devices in a cognitive network must register their locations in the database. In addition, fixed devices must transmit identifying information to make it easier to identify them if they are found to interfere. Furthermore, fixed and personal/portable devices operating independently must provide identifying information to the TV bands database. However, FCC permits applications for certification of devices that do not include the geo-location and database access capabilities and instead rely on spectrum sensing to avoid causing harmful interference. A fixed device must employ both geo-location, database access, and spectrum sensing capabilities that enable the device to listen for and identify the presence of signals from other transmitters. A personal/portable device must either be under the control of a fixed device or a personal/portable device that employs geo-location, database access and spectrum sensing or employ geo-location/database access and spectrum sensing itself. Therefore, the assumption of a clustered wireless network, in which each cluster consists of a number of users is very reasonable in the context of cognitive networks sharing white spaces horizontally. 
Another example is the homogeneous/heterogeneous clustered sensor networks for target tracking and in general, anomaly detection purposes [9], [11], [39]. In such networks, agile clusters are formed which comprise pressure, sonar and magnetic sensors. Each cluster is equipped with a cluster-head, which communicates with the cluster members, gathers their data and reports to a fusion center. Often times a number of such clusters coexist in the same space-time neighborhood, and hence spectrum sharing is very desired in order to increase the throughput of the underlying intra-cluster communication links.

Further examples include Wireless LAN Hotspots and WPAN networks, in which intra-cluster communication is very desired. There are a number of efficient methods for partitioning the network elements, which will lead to such clustered networks. However, these methods are not the focus of this paper and we are assuming that the clusters are already formed in a specified manner.

Digital Subscriber Lines: In a Digital Subscriber Line (DSL) system, modems use frequencies above the voice band for handling high-speed data. The bundle of transmission lines may contain up to 100 subscriber lines. Clearly, there will be electromagnetic interference between the lines in the bundle. Thus, the DSL system can be modeled as a number of transmitters and receivers interfering into each other [58].

Thus, for a clustered wireless network, each node is a collection of users forming a cluster and in a DSL system each node is a subscriber line. In the former case, each node has a cluster-head responsible for managing some of the network functions. The canonical network model is given by a collection of nodes, $c_{i}, i=1, \cdots, N$, which is depicted in Fig. 1 for a clustered wireless network.

Remark: Bambos [7] has introduced a canonical conceptual framework for the networking paradigm, in which the network is conceptually modeled as a collection of interfering links. According to [7], two instances of this concept are ad hoc networks (with no fixed infrastructure and possibly multi-hop communication mode) and cellular networks (with a fixed infrastructure and single-hop communication mode). The canonical network model of this paper can also be viewed as a collection of interfering links. However, the two network models pertain to different structural scales; the canonical network model in our paper consists of a number of co-existing and hence interfering network nodes, where each node may have an internal structure à al the 


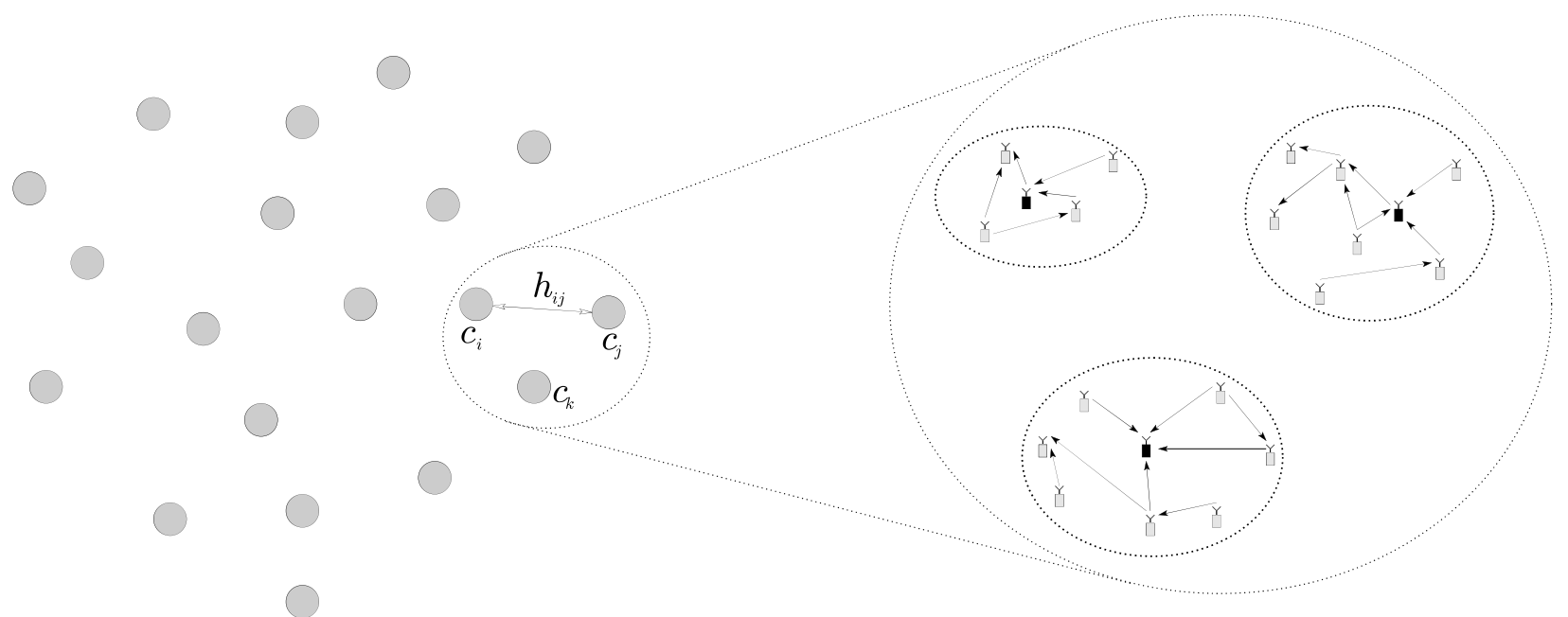

Fig. 1. Canonical Network Model

canonical conceptual model of Bambos [7]. For example, in case of clustered wireless networks, the network consists of a number of co-existing clusters, where each cluster comprises a number of users along with a cluster-head. On a smaller scale, the users in each cluster may form a network of the type described in [7], e.g., the users inside a cluster may communicate with each other or the cluster-head in a multi-hop/single-hop communication mode. The case of the DSL network is slightly different: a DSL network consists of a bundle of co-existing and hence interfering wires, but the wires have no internal network structure.

\section{A. Assumptions}

We make the following main assumptions on the network model:

1) The interference between any two nodes is reciprocal.

2) The leakage interference between any two nodes is symmetric.

3) The node $c_{i}$ transmits with power $P_{i}$.

4) The channel between nodes $c_{i}$ and $c_{j}$ is given by $h_{i j}$. For example, $h_{i j}$ can be modeled as a Rayleigh fading channel or path loss with exponent $\eta$. We also denote the self-gain of a node by $h_{i i}$.

5) The accessible spectrum is divided into $r$ different bands, denoted by $b_{1}, \cdots, b_{r}$. 
6) At time $t$, the $i$ th node is in state $s_{i}(t) \in\{1,2, \cdots, r\}$, corresponding to the index of the frequency band it is using for communication purposes.

7) The rate of change of the spatial distributions of the nodes in the network is much less than the processing/transmission rate. Therefore, the topology of the network is assumed to be fixed in the analysis of the frequency allocation algorithm.

Remark: Assumption 1 readily holds for the DSL network due to the physical properties of transmission lines. In other words, in the DSL network $h_{i i}$ is simply the self-inductance of the $i$ th wire (in contrast to $h_{i j}$ which is the mutual inductance of wires $i$ and $j$ ). Hence, interference reciprocity is implied by the mutual inductance reciprocity, since the self-gain $h_{i i}$ is typically the same for all wires.

For clustered wireless networks in general, there are various ways in order to obtain interference symmetry, which are discussed in details in [35]. In a more specific network scenario, where the mutual distances between the clusters in a clustered wireless network are much larger than the typical cluster size, one can obtain interference symmetry from channel reciprocity by adding the additional assumption that at each moment in each cluster there is at most one user transmitting. In this case, $h_{i j}$ can be interpreted as the link gain between the transmitting user in cluster $i$ and $j$, and $h_{i i}$ can be interpreted as the typical link gain between the cluster-head and cluster members of cluster $i$. This assumption can be enforced by employing a TDMA scheme for intra-cluster communication [26], or considering the 802.11e or 802.16h scenarios. We further assume that the clusters have similar physical characteristics, hence $h_{i i}=h_{j j}$ for all $i$ and $j$.

\section{B. Network Utility}

A common choice for the network utility is the sum-rate (See, for example, [13], [15], [58]). Let node $c_{i}$ be in state $s_{i}$, i.e., transmitting in frequency band $b_{s_{i}}$. We define the rate of a node as follows:

$$
R_{i}:=\log \left(1+\frac{P_{i}}{N_{0}\left(s_{i}\right)+I_{c_{i}}}\right)
$$

where $N_{0}\left(s_{i}\right)$ is the noise power in band $s_{i}$ and $I_{c_{i}}$ is the interference experienced by $c_{i}$. The interference experienced by node $c_{i}$, given the state of the system being $\left\{s_{1}, s_{2}, \cdots, s_{N}\right\}$, can 
be modeled as

$$
I_{c_{i}}:=\sum_{j \neq i} P_{j} \alpha_{i j} f\left(s_{i}, s_{j}\right)
$$

where

$$
\alpha_{i j}:=\frac{h_{i j}}{h_{i i}}
$$

and $f\left(s_{i}, s_{j}\right)$ denotes the symmetric leakage interference of the frequency band $s_{j}$ into $s_{i}$. For example, if the leakage between different frequency bands is negligible, $f$ can be well approximated by the Kronecker delta function:

$$
\delta\left(s_{i}, s_{j}\right)= \begin{cases}1 & s_{i}=s_{j} \\ 0 & s_{i} \neq s_{j}\end{cases}
$$

As mentioned in Section II-A, we assume that $h_{i i}$ is constant across different nodes in the network, hence the condition of interference symmetry, i.e., $\alpha_{i j} f\left(s_{i}, s_{j}\right)=\alpha_{j i} f\left(s_{j}, s_{i}\right)$, is implied by $h_{i j}=h_{j i}$, which is the channel reciprocity between nodes $i$ and $j$.

The sum-rate can then be defined as

$$
U^{\text {sum-rate }}:=\sum_{i=1}^{N} R_{i}
$$

The network utility function considered in this paper is the weighted aggregate interference and is defined as

$$
U:=-\sum_{i=1}^{N} P_{i} I_{c_{i}}
$$

The weight of the interference experienced by node $c_{i}$ is given by its transmission power $P_{i}$. A similar metric has been introduced by Lacatus et al. [28] in the context of power and codeword adaptation in CDMA systems. It is possible to consider a more general network utility for the case where the nodes allocate their total power across the $r$ frequency bands. Let $P_{i}^{k}$ be the transmission power of node $c_{i}$ in frequency band $b_{k}$, such that

$$
\sum_{k=1}^{r} P_{i}^{k}=P_{i} .
$$

We can define a similar power-weighted aggregate interference as follows:

$$
U^{\prime}:=-\sum_{i=1}^{N} \sum_{k=1}^{r} P_{i}^{k} I_{c_{i}}^{k}
$$


where the additional summation over $k$ captures the effect of power distribution across all the frequency bands. As it will be discussed later in Section III-A, maximizing the network utility $U^{\prime}$ reduces to maximizing the network utility $U$ (Further motivations for choosing such a network utility function is discussed in detail in Section III-A). Therefore, we will only present our analysis for the network utility $U$.

Other metrics such as aggregate SIR (See Sung et al. [51]) and log-sum-rate (See Etkin et al. [15]) have also been studied in the literature. Finally, note that the entire analysis throughout this paper can be carried out by negating the utility function $U$ and considering utility minimization. This way, the network utility will be a positive quantity and the results (especially the inequalities) will be more intuitive. However, in order to align ourselves to the common notion of utility maximization, we prefer to work with negative utility functions in favor of maintaining utility maximization as our objective.

\section{Connection of the Weighted Aggregate Interference to Sum-rate}

The negated weighted aggregate interference has been used as a successful metric in some strands of results in the existing literature (See, for example, [28], [34], [35] and [36]). However, we find it useful to delineate the connection of this metric to the sum-rate metric. In what follows, we show that there exists a regime of SINR (i.e., low SINR regime) where maximizing the negated aggregate interference yields an approximate solution to the maximization of the sum-rate. The low SINR regime is defined by the following set of conditions:

$$
\alpha_{i}:=\frac{P_{i}}{N_{0}\left(s_{i}\right)+I_{c_{i}}} \ll 1
$$

for all $i=1,2, \cdots, N$. We also assume that $N_{0}\left(s_{i}\right) \ll I_{c_{i}}$ for all $i=1,2, \cdots, N$ and therefore focus our attention on the low SIR regime. A practical example of a network with low SIR is the CDMA/HDR network. In fact, several measurements done by Bender et al. [10] reveal that almost $50 \%$ of the nomadic users in a CDMA/HDR system have negative SIR (in the dB scale). In this regime, the sum-rate can be approximated by

$$
U^{\text {sum-rate }}=\sum_{i} \frac{P_{i}}{I_{c_{i}}}+\mathcal{O}\left(\left\{\alpha_{i}^{2}\right\}\right),
$$

since $\log (1+x) \approx x$ for $x \ll 1$. Moreover, if we assume that the network is homogeneous, i.e., the nodes have very similar structures (which is true for DSL and clustered wireless networks) 
and the number of interferers and available frequency bands are large enough (See Remark 1 below), the interference experienced by node $c_{i}$ can be expressed as

$$
I_{c_{i}}=\bar{I}+\delta I_{c_{i}}
$$

with $\beta_{i}:=\delta I_{c_{i}} / \bar{I} \ll 1$ for all $i=1,2, \cdots, N$. Note that $\bar{I}$ captures the typical interference experienced by the nodes in the homogeneous network and $\delta I_{c_{i}}$ stands for the variations in the interference levels. The sum-rate can be then further approximated by

$$
\begin{aligned}
U^{\text {sum-rate }} \approx \sum_{i} \frac{P_{i}}{\bar{I}}\left(1-\frac{\delta I_{c_{i}}}{\bar{I}}\right) & =\sum_{i} \frac{P_{i}}{\bar{I}}\left(2-\frac{I_{c_{i}}}{\bar{I}}\right) \\
& =U_{0}+\frac{1}{\bar{I}^{2}} U
\end{aligned}
$$

where

$$
U_{0}:=2 \sum_{i} \frac{P_{i}}{\bar{I}}
$$

independent of $\delta I_{c_{i}}$, for $i=1,2, \cdots, N$, and the overall error is of the order

$$
\mathcal{O}\left(\left\{\alpha_{i}^{2}\right\}\right)+\mathcal{O}\left(\left\{\beta_{i}^{2}\right\}\right)
$$

Therefore, $U^{\text {sum-rate }} \approx U_{0}+\frac{1}{I^{2}} U$ and maximizing $U$ is equivalent to maximizing $U^{\text {sum-rate }}$ for a homogeneous network in low SIR regime (Note that a similar affine relation holds between $U^{\text {sum-rate }}$ and $U^{\prime}$ ). Simulation studies in Section VI verify that the maximization of the negated aggregate interference indeed yields a near-optimal solution to sum-rate maximization.

Remark: The hypothesis of $\delta I_{c_{i}} \ll \bar{I}$ is adopted from statistical physics. If the network geometry is homogenous with high number of interferers, and there is a considerable number of available frequency bands, this condition holds for generic spatial and frequency band configurations of the network nodes. As an example, let us consider a scenario where the nodes are distributed in the plane with a density of $n_{0}$ nodes per normalized unit area. Also, we suppose that each node can take any of the $r$ available frequency bands with equal probability. It is easy to show that the ratio of standard deviation to mean of the interference for each user scales as $\sqrt{(r-1) / n_{0}}$. Hence, if $n_{0} \gg r-1$, the hypothesis holds with high probability for generic spatial configurations of the network nodes with random frequency band assignments. In the jargon of statistical physics, this corresponds to the high temperature behavior of the system, where each state is accessible equi-probably. It is worth considering the low temperature behavior, where 
the network lives near the optimal frequency band assignment (i.e., equilibrium, in the statistical physics terms). In this case, the most probable states of the system occur when one node switches away from the optimal frequency band to a non-optimal band with equal probability. In is easy to show that the variations in the interference experienced by each user scale as $O(1 / r)$. Hence, if $r \gg 1$, the hypothesis holds for generic configurations of the system near the optimal frequency band assignment. Therefore, when both $n_{0} \gg r-1$ and $r \gg 1$ are satisfied, the assumption of $\delta I_{c_{i}} \ll \bar{I}$ is reasonable for generic spatial and frequency band configurations of the network nodes.

\section{Discrete-time vs. Continuous-time Models}

Let the state of the network be $\left\{s_{1}(t), s_{2}(t), \cdots, s_{N}(t)\right\}$ at time $t$. Each node, say $c_{i}$, picks a time $t=t_{n}$ at random and updates its transmission frequency band. The nature of the update procedure is asynchronous for all the nodes. This is intuitively appealing, because of the nature of distributed networks, where there is usually no common clock among the nodes. We assume that the updates are taking place at times $t=t_{1}, t_{2}, \cdots$. The update process can be different for each node. For example, each node can choose the update times based on a point process (e.g., Poisson point process) with a specific rate. The update process will be discussed in more detail in Section V. In this case, we can express the interference experienced by node $c_{i}$ at time $t$ by

$$
I_{c_{i}}(t):=\sum_{\substack{j=1 \\ j \neq i}}^{N} P_{j} \alpha_{i j} f\left(s_{i}(t), s_{j}(t)\right)
$$

The network utility at time $t, U(t)$, can be written as

$$
U(t):=-\sum_{i=1}^{N} P_{i} I_{c_{i}}(t)=-\sum_{i=1}^{N} \sum_{\substack{j=1 \\ j \neq i}}^{N} P_{i} P_{j} \alpha_{i j} f\left(s_{i}(t), s_{j}(t)\right)
$$

Let $G_{k}(t) \subseteq\{1,2, \cdots, N\}$ denote the set of nodes transmitting in band $b_{k}$ at time $t$. Also, let $I_{c_{i}}^{k}(t)$ denote the interference experienced by $c_{i}$ caused by all the nodes in $G_{k}(t)$, if $c_{i}$ was transmitting in band $b_{k} . I_{c_{i}}^{k}(t)$ can be written as

$$
I_{c_{i}}^{k}(t)=\sum_{\substack{j=1 \\ j \neq i}}^{N} P_{j} \alpha_{i j} f\left(k, s_{j}(t)\right)
$$


Whenever the temporal dynamics of the updates are not important for our purposes, we can alternatively use a discrete-time state-space model, where the state of system is given by $\left\{s_{1}[n], s_{2}[n], \cdots, s_{N}[n]\right\}$ at time $n$, corresponding to $t=t_{n}$. The quantities $I_{c_{i}}[n], U[n]$ and $I_{c_{i}}^{k}[n]$ can be similarly defined by substituting the continuous-time states $s_{1}(t), s_{2}(t), \cdots, s_{N}(t)$ with their discrete-time versions, $s_{1}[n], s_{2}[n], \cdots, s_{N}[n]$.

It must be noted that, for notational convenience, we may drop the time dependence of the functions $I_{c_{i}}[n], I_{c_{i}}^{k}[n]$ and $U[n]$ following the convergence of the algorithm or whenever the state of the system is not varying over time, and denote them by $I_{c_{i}}, I_{c_{i}}^{k}$ and $U$, respectively. Also, we may add the dependence on $N$, the number of nodes, upon convenience and denote the above functions by $I_{c_{i}}(N), I_{c_{i}}^{k}(N)$ and $U(N)$, respectively.

\section{The GADIA ALgorithm}

Given the canonical network model and the utility function $U$ in Section II, the objective is to design a distributed algorithm to maximize the network utility function across different nodes in the network. Note that each node, say $c_{i}$, can only observe $I_{c_{i}}^{k}[n]$ for all $k=1,2, \cdots, r$. For example, in a clustered wireless network, the cluster-head scans the spectrum and estimates/measures the interference it experiences in each frequency band. Thus, each node is only aware of its own interference profile. Given the measured interference profile, each node needs to update its frequency band in a way to increment the network utility. Our proposed distributed algorithm for this purpose is called the Greedy Asynchronous Distributed Interference Avoidance (GADIA) Algorithm. We can formally define the GADIA algorithm as follows:

The GADIA Algorithm: Nodes scan all the frequency bands $b_{1}, \cdots, b_{r}$ in an asynchronous manner over time. Each node chooses the frequency band in which it experiences the least interference from other nodes. In other words, a node $c_{i}$ picks a time $n$ at random and updates its state according to the following rule

$$
s_{i}[n+1]=\arg \min _{j} I_{c_{i}}^{j}[n] .
$$

If the minimizer is not unique, $c_{i}$ randomly picks one such minimizer. 


\section{A. Discussions}

In case of clustered wireless networks, the cluster-head chooses the new transmission frequency band according to Eq. (18), and then announces the new frequency band to the other users in the cluster. In case of a DSL system, the interference channels can be measured in the loop-planning phase and then the transmitter modems can shape their PSD according to the GADIA algorithm. We will mainly focus on the clustered wireless networks throughout the paper, and will point out the relevant analogies to DSL systems whenever convenient.

As confirmed by simulations in Section VI, such a network utility introduces more robustness to the performance of the distributed frequency allocation algorithm in presence of timevariations, in comparison with the Iterative Water-filling algorithm [58]. Moreover, the GADIA algorithm achieves a higher sum-rate compared to the Iterative Water-filling algorithm in the low SIR regime (See Section VI). Finally, as it will be discussed in the forthcoming sections, our choice of network utility admits a tractable mathematical framework to analyze the performance of the GADIA algorithm under time-variations (see Section V) and uncertainties [2].

The constraint that each node chooses a single frequency band for communication purposes at each time is consistent with the network utility $U$. In fact, this is induced by this particular choice of network utility. In contrast, suppose that each node can allocate its total power across the $r$ available frequency bands. Consider the network utility $U^{\prime}$ adapted to this new power allocation strategy, defined in Eq. (8) as:

$$
U^{\prime}=-\sum_{i=1}^{N} \sum_{k=1}^{r} P_{i}^{k} I_{c_{i}}^{k}
$$

It is easy to check that $U^{\prime}$ has an affine relation with $U^{\text {sum-rate }}$ as in Eq. (12). The node $c_{i}$ can only observe $I_{c_{i}}^{k}, k=1,2, \cdots, r$ and can allocate its total power $P_{i}$ across the $r$ frequency

bands in order to increment the network utility. Let $P_{i}^{k}$ be the transmission power of node $c_{i}$ in frequency band $b_{k}$, such that

$$
\sum_{k=1}^{r} P_{i}^{k}=P_{i}
$$

Then, the node $c_{i}$ needs to solve the following local optimization problem:

$$
\max _{\left\{P_{i}^{k}\right\}_{k=1}^{r}}\left\{-\sum_{k} P_{i}^{k} I_{c_{i}}^{k}\right\} \text { subject to } \sum_{k} P_{i}^{k}=P_{i}
$$


Since the objective function is linear in $P_{i}^{k}$, clearly the solution is to allocate the total power $P_{i}$ to the frequency band $b_{k^{*}}$ such that

$$
k^{*}=\arg \min _{k} I_{c_{i}}^{k} .
$$

Therefore, even if the nodes start the update process with power allocation across all the frequency bands, the above choice of power-weighted aggregate interference for the network utility will give rise to single frequency band allocations in the steady state. In light of the above, we only analyze the algorithm under the network utility $U$, corresponding to the single frequency band assignments.

In the Iterative Water-filling algorithm [58], node $c_{i}$ allocates its power across the frequency bands as follows:

$$
P_{i}^{j}=\left(\nu-I_{c_{i}}^{j}\right)_{+}
$$

where $(x)_{+}:=\max (x, 0)$ and $\nu$ is selected such that

$$
\sum_{j=1}^{r}\left(\nu-I_{c_{i}}^{j}\right)_{+}=P_{i}
$$

First of all, we note that the Iterative Water-filling algorithm clearly has higher computational complexity compared to the GADIA algorithm. Secondly, as mentioned in the introduction, the outcome of the Iterative Water-filling algorithm might not always be near-optimal. In fact, as pointed out by Etkin et al. [15] and O. Popescu et al. [42], the outcome of the Iterative Waterfilling algorithm might be the only possible outcome and far from optimal. For example, let us consider the scenario in Fig. 3. There are two nodes, $c_{1}$ and $c_{2}$, and two available frequency bands, $b_{1}$ and $b_{2}$. The initial power allocation is the flat power allocation across the two frequency bands. Clearly, this is an equilibrium point for the Iterative Water-filling algorithm, since the best response of each node to the flat power allocation of the other is the flat allocation allocation [15], [42]. On the other hand, the GADIA algorithm bypasses such a Nash equilibrium, due to its inherent equilibrium selection via tie-breaking. In other words, the GADIA algorithm chooses a single frequency band, even if several available frequency bands have the same interference level. Hence, GADIA would indeed quickly segregate itself onto an equilibrium in which the two nodes do not impose any interference on each other. Thus, for this example, the output of the GADIA algorithm has clearly a higher sum-rate than that of the Iterative Water-filling. 

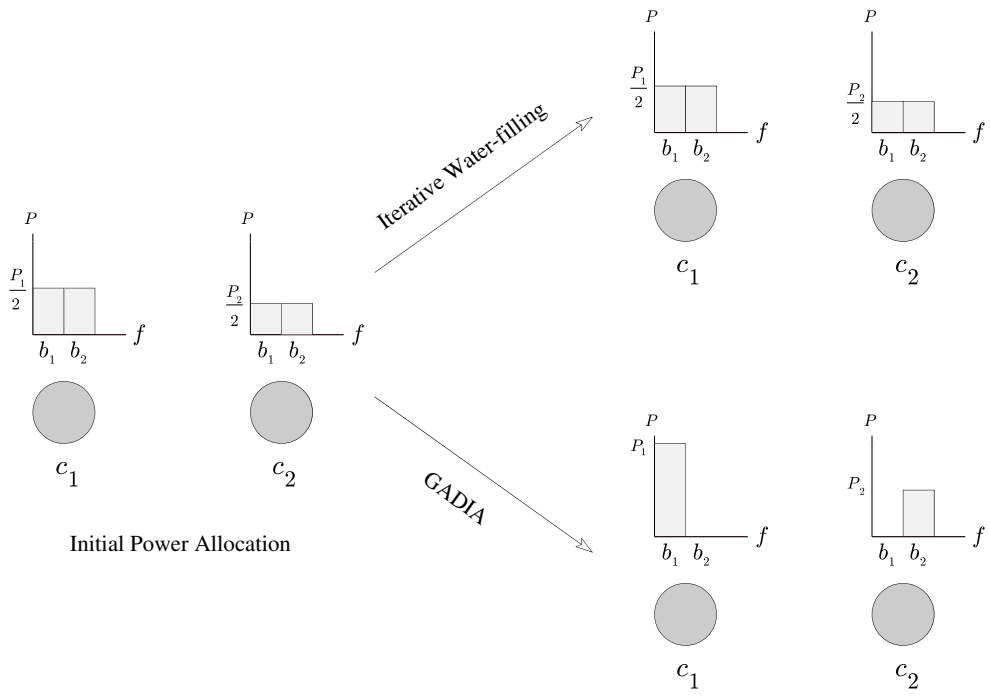

Fig. 2. The equilibrium of Iterative Water-filling vs. GADIA; GADIA results in full interference avoidance, whereas Iterative Water-filling flattens the PSD.

Although this example is not very generic, it captures the essence of difference between the two algorithms. In particular, in the low SIR regime, the GADIA algorithm is more likely to output a frequency band assignment with significant interference avoidance, compared to Iterative Water-filling (since in this regime, the Iterative Water-filling algorithm tends to flatten the spectrum).

Also, in clustered wireless networks, the clusters may be very close to each other and even partially overlapping: For example, in a cognitive ad hoc network, it is possible to have partially overlapping clusters of users which are communicating with a few fixed devices equipped with geo-location systems. Moreover, due to the nature of the wireless channel and also high number of interferers, the SIR at the receivers might be very low. For example, in CDMA/HDR systems almost $50 \%$ of the nomadic users suffer from negative SIR (in the dB scale) [10]. Therefore, in general, one expects the network to be operating in a relatively low SIR environment. This is not usually the case in the DSL networks, since the subscriber lines can not physically overlap. Simulation results in Section VI confirm that the GADIA algorithm outperforms the Iterative Water-filling algorithm in a relatively low SIR regime, for a broad range of clustered wireless networks with different topologies. 


\section{Main Results: Static Analysis}

First, we will establish the convergence of the GADIA algorithm under the hypothesis of interference reciprocity. Then, we will present performance bounds for the GADIA algorithm under different network topologies.

\section{A. Convergence}

Theorem 4.1: Given any reciprocal interference model, the GADIA algorithm converges to a local minimum.

Proof: First, we show that $-U[n]$ is a non-increasing function of $n$. Without loss of generality, we assume that the node $c_{k}$ is updating its frequency band at time $n$. Suppose that $c_{k}$ has been transmitting in band $b_{s_{k}[n]}$ at time $n$. Thus, $U[n]$ can be written as

$$
\begin{aligned}
U[n]= & -\sum_{\substack{i, j=1 \\
i \neq j \neq k}}^{N} P_{i} P_{j} \alpha_{i j} f\left(s_{i}[n], s_{j}[n]\right)-\sum_{i=1, i \neq k}^{N} P_{i} P_{k} \alpha_{i k} f\left(s_{i}[n], s_{k}[n]\right) \\
& -\sum_{i=1, i \neq k}^{N} P_{k} P_{i} \alpha_{k i} f\left(s_{k}[n], s_{i}[n]\right) .
\end{aligned}
$$

Since, $\alpha_{i k}=\alpha_{k i}$, for all $i \neq k$ by the assumption of interference reciprocity and $f\left(s_{i}, s_{k}\right)=$ $f\left(s_{k}, s_{i}\right)$ by the leakage symmetry hypothesis, we can write $U[n]$ as

$$
\begin{aligned}
U[n] & =-\sum_{\substack{i, j=1 \\
i \neq j \neq k}}^{N} P_{i} P_{j} \alpha_{i j} f\left(s_{i}[n], s_{j}[n]\right)-2 \sum_{i=1, i \neq k}^{N} P_{i} P_{k} \alpha_{i k} f\left(s_{i}[n], s_{k}[n]\right) \\
& =-\sum_{\substack{i, j=1 \\
i \neq j \neq k}}^{N} P_{i} P_{j} \alpha_{i j} f\left(s_{i}[n], s_{j}[n]\right)-2 P_{k} I_{c_{k}}^{s_{k}[n]}[n]
\end{aligned}
$$

After the update, $c_{k}$ chooses the new band $b_{s_{k}[n+1]}$, where

$$
s_{k}[n+1]=\arg \min _{j} I_{c_{k}}^{j}[n] .
$$

Therefore,

$$
\begin{aligned}
U[n+1] & =-\sum_{\substack{i, j=1 \\
i \neq j \neq k}}^{N} P_{i} P_{j} \alpha_{i j} f\left(s_{i}[n], s_{j}[n]\right)-2 P_{k} I_{c_{k}}^{s_{k}[n+1]}[n] \\
& =U[n]-2 P_{k}\left(I_{c_{k}}^{s_{k}[n+1]}[n]-I_{c_{k}}^{s_{k}[n]}[n]\right)
\end{aligned}
$$


Eq. (27) implies that $I_{c_{k}}^{s_{k}[n+1]}[n] \leqslant I_{c_{k}}^{s_{k}[n]}[n]$. Hence,

$$
U[n+1]-U[n] \geqslant 0
$$

which implies that $-U[n]$ is a non-increasing function of $n$. Moreover, $-U[n]$ is clearly lower bounded by 0 . Therefore, $\exists M \in \mathbb{N}$ such that $\forall m \geqslant M$, we have $U[n+1]=U[n]$, which proves the statement of the Theorem.

\section{B. Performance Bounds: Lower Bound}

Theorem 4.2 gives a lower bound on the performance of the GADIA algorithm for the general canonical network model.

Theorem 4.2 (Lower Bound): Let $U_{G}$ denote the network utility corresponding to the state of the algorithm following convergence (see Theorem 4.1), and $U_{w}$ be the network utility corresponding to the worst case interference scenario (where all nodes transmit in the same frequency band). Then,

$$
U_{G} \geqslant \frac{1}{r} U_{w}
$$

where $r$ is the number of available frequency bands.

Proof: Suppose that at time $n$ the node $c_{i}$ chooses the frequency band $b_{k}$. Therefore, we have $I_{c_{i}}^{k}[n] \leqslant I_{c_{i}}^{j}[n]$, for all $j \neq k$. Hence,

$$
r I_{c_{i}}^{k}[n] \leqslant \sum_{j=1}^{r} I_{c_{i}}^{j}[n]
$$

Note that the right-hand side is independent of $n$, since it stands for the interference experienced by $c_{i}$ when all the other nodes are transmitting in the same frequency band. Let $M$ be a time following the convergence of the GADIA algorithm. Clearly, the above inequality holds for time $M:$

$$
r I_{c_{i}}^{k}[M] \leqslant \sum_{j=1}^{r} I_{c_{i}}^{j}[M]
$$

and for all nodes $c_{i}, i=1,2, \cdots, N$. On the other hand, $U_{w}$ can be written as

$$
U_{w}=-\sum_{i=1}^{N} \sum_{j=1}^{r} I_{c_{i}}^{j}[M]
$$


Summing over $i$ on the both sides of Eq. (32) yields:

$$
-r U_{G}=r \sum_{i=1}^{N} I_{c_{i}}^{k}[M] \leqslant \sum_{j=1}^{r} I_{c_{i}}^{j}[M]=-U_{w}
$$

which proves the statement of the theorem.

Note that so far the network model under study is the canonical model in its most general form. In order to obtain concrete performance bounds, we will make a number of simplifying assumptions on the network topology, power constraints and channel model to make the mathematical analysis tractable. In particular, we focus our attention to clustered wireless networks and assume that the channel model is path loss with exponent $\eta$, i.e.,

$$
h_{i j}=\frac{1}{d_{i j}^{\eta}}
$$

where $d_{i j}$ is the distance between nodes (clusters) $c_{i}$ and $c_{j}$. We further assume that the quantities $d_{i j}$ are normalized by the size of the clusters, so that

$$
\alpha_{i j}=\frac{1}{d_{i j}^{\eta}}
$$

Furthermore, we assume that the leakage between different frequency bands is negligible and approximate the leakage function $f\left(s_{i}, s_{j}\right)$ by the Kronecker delta function, $\delta\left(s_{i}, s_{j}\right)$, as discussed before in Section II-B. When all the clusters have the same size, i.e., homogeneous network, $h_{i i}$ is constant across different nodes. Thus, the model is interference reciprocal. Therefore, all the previous results (convergence and lower bound) hold. Under the foregoing assumptions, the network utility at time $n$ takes the following form:

$$
U[n]:=-\sum_{\substack{i, j=1 \\ i \neq j}}^{N} \frac{P_{i} P_{j}}{d_{i j}^{\eta}} \delta\left(s_{i}[n], s_{j}[n]\right)
$$

Remark: Note that the asymptotic behavior of $\alpha_{i j}$ must be such that $U[n] / N$ is well-defined for any choice of state variables $s_{i}[n]$, as $N \rightarrow \infty$. In particular, for the path loss model with exponent $\eta$, we must have $\eta>D$, where $D$ is the dimension of the space over which the nodes are distributed. 


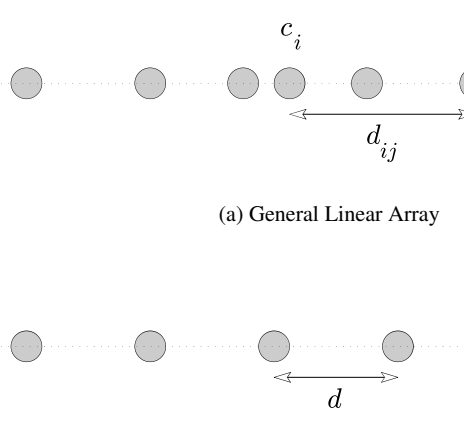

(b) Uniform Linear Array

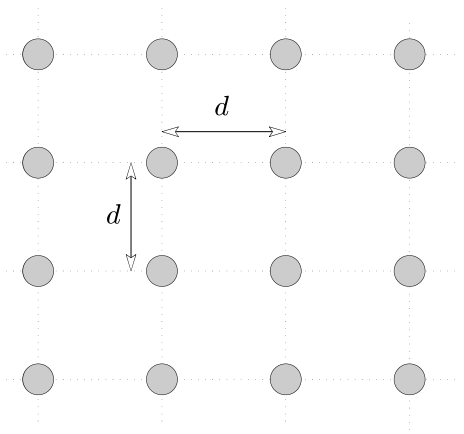

(c) Uniform 2D Array

Fig. 3. Examples of network topologies. a) General linear array, b) Uniform linear array, and c) Uniform 2D array.

\section{Performance Bounds: Asymptotic Upper Bound}

It is in general hard to characterize the optimal frequency band assignment, even for simple network topologies such as linear arrays. This is due to the fact that nodes have long-range interactions, which makes the centralized network utility maximization problem non-trivial. One simplification is to consider short-range interactions, e.g., nearest neighbors, which is widely studies in Statistical Physics in the context of Ising-type models (See, for example, [8]). Also, a graph model has been studied by Peng et al. [38], which fairly simplifies the interference model. In light of the above, in order to compare the performance of the GADIA algorithm to the optimal centralized strategy, we will obtain a non-trivial lower bound on the optimal network utility.

Fig. 3 shows some examples of the network topologies studied in this paper. The nodes forming a linear array are shown in Fig. 3a. For a given $N$, we assume that the nodes are located in $[0,(N-1) d]$, where $d$ is a constant. As a special case, when each node is a distance $d$ apart from its neighbors, we denote the array by uniform linear array (Fig. 3b). Note that the uniform linear array corresponds to the integer $\mathbb{Z}_{1}$ lattice [14]. Generalizations of the uniform linear array to higher dimensions, which are appropriate for our purposes, are referred to as Bravais lattices (See, for example, [14]). For example, one such lattice in 2 dimensions is the uniform $2 D$ array shown in Fig. 3c, which corresponds to the integer $\mathbb{Z}_{2}$ lattice [14]. Before moving on to the main 
result regarding the lower bound, we mention our motivation for employing Bravais lattices and briefly review some of their properties.

Let $U_{o}\left(N^{D}\right)$ be the network utility corresponding to the optimal strategy for a given spatial distribution of $N^{D}$ nodes inside the $D$ dimensional cube of side $N d$. Recall that $G_{k} \subseteq\{1,2, \cdots, N\}$ is the subset of nodes transmitting in frequency band $b_{k}$, corresponding to the optimal frequency band assignment. Let $N_{k}:=\left|G_{k}\right|$. We express $U_{o}\left(N^{D}\right)$ as follows:

$$
U_{o}\left(N^{D}\right)=\sum_{k=1}^{r} U_{w}\left(N_{k}\right)
$$

where $U_{w}\left(N_{k}\right)$ denotes the utility corresponding to the $N_{k}$ nodes in the set $G_{k}$, which are all transmitting in band $b_{k}$ (hence the subscript $w$ ).

Let us consider the nodes in $G_{k}$ and suppose that they are particles inside the $D$ dimensional cube of side $N d$, interacting with one another according to the following vector field:

$$
\mathbf{F}_{i j}=\frac{P_{i} P_{j}}{\eta d_{i j}^{\eta+2}}\left(\mathbf{x}_{i}-\mathbf{x}_{j}\right),
$$

where $\mathbf{x}_{i}$ denotes the position of node $c_{i}$ in space. This interaction field is clearly repulsive. Therefore, if we arbitrarily distribute the nodes inside the $D$ dimensional cube of side $N d$ (with the freedom to move inside the cube), the final equilibrium configuration is when the potential function given by

$$
V_{G_{k}}:=\sum_{i \in G_{k}} \sum_{\substack{j \in G_{k} \\ j \neq i}} \frac{P_{i} P_{j}}{d_{i j}^{\eta}}
$$

achieves a local minimum. We note that the potential function $V_{G_{k}}$ is exactly equal to $-U_{w}\left(N_{k}\right)$. Therefore, the final configuration of the nodes is when the network utility corresponding to the nodes in $G_{k}$ is maximized. Let $U_{w}^{*}\left(N_{k}\right)$ denote the network utility of $N_{k}$ nodes which are distributed in space according the spatial configuration corresponding to the global minimum of the potential function $V_{G_{k}}$. We then have $U_{w}\left(N_{k}\right) \leqslant U_{w}^{*}\left(N_{k}\right)$. This inequality is the key to proving the main theorem regarding the optimal frequency band assignment (Theorem 4.3).

If $P_{i}=P_{0}$ for all $i=1,2, \cdots, N$, the configuration corresponding to any local minimum of the above potential function, in the limit of $N \rightarrow \infty$, is assumed to be a $D$ dimensional Bravais lattice. For example, all the natural crystals are formed by repeatedly placing a collection of 
atoms on a Bravais lattice. Bravais lattices are well-studied in the context of crystallography and solid state physics (See, for example, [1]). For example, it is shown that there are 1, 5 and 14 different Bravais lattices in 1,2 and 3 dimensions, respectively. The unique Bravais lattice in 1 dimension is simply the integer $\mathbb{Z}_{1}$ lattice.

Although in solid state physics it is widely assumed that the configuration corresponding to the minimum of the potential function of particles interacting via an isotropic and convex repulsive field is a Bravais lattice, a mathematically rigorous proof in 2 and 3 dimensions does not exist. A recent article [52] addresses construction of such proofs for a class of interactions which include those with power law asymptotics (e.g., the path loss model with exponent $\eta$ ). The proof for the 1 dimensional case is given in [54].

Let $\mathcal{L}(d)$ be a Bravais lattice in $D$ dimensions with unit spacing $d$ and generators $\mathbf{a}_{1}, \mathbf{a}_{2}, \cdots, \mathbf{a}_{D}$ [14]. We define the energy of the lattice $\mathcal{L}(d)$ as follows:

$$
E(\eta):=\sum_{\substack{i_{1}, i_{2}, \cdots, i_{D}=-\infty \\\left(i_{1}, i_{2}, \cdots, i_{D}\right) \neq 0}}^{\infty} \frac{1}{\left\|i_{1} \mathbf{a}_{1}+i_{2} \mathbf{a}_{2}+\cdots+i_{D} \mathbf{a}_{D}\right\|_{2}^{\eta}}
$$

For example, for $D=1$, the Bravais lattice (integer $\mathbb{Z}_{1}$ lattice) has energy $E(\eta)=\zeta(\eta)$, the Riemann zeta function. The values of $E(\eta)$ for different Bravais lattices can be easily computed numerically. Let $\mathcal{L}^{*}(d)$ be the $D$ dimensional Bravais lattice with minimum energy $E_{\min }(\eta)$. The alternating frequency band assignment on the $\mathcal{L}^{*}(d)$ lattice can be defined as assigning frequency bands $b_{1}, b_{2}, \cdots, b_{r}$ to the $r$ cosets of $\mathcal{L}^{*}(d)$, with unit spacing $r^{1 / D} d$, respectively. The alternating frequency band assignment is commonly used in cellular communication systems (See, for example, [31] and [44]) as the optimal frequency reuse strategy.

Theorem 4.3 gives a non-trivial upper bound on the optimal network utility of a $D$ dimensional network:

Theorem 4.3 (Optimal Utility In D Dimensions): Let $U_{o}\left(N^{D}\right)$ be the network utility corresponding to the optimal strategy for a given spatial distribution of $N^{D}$ nodes inside the $D$ dimensional cube of side $N d$. Moreover, assume that $P_{j}=P_{0}$ for all $j=1,2, \cdots, N$. Then,

$$
\lim _{N \rightarrow \infty} \frac{1}{N^{D}} U_{o}\left(N^{D}\right) \leqslant-\frac{1}{r^{\frac{n}{D}}} E_{\min }(\eta) \frac{P_{0}^{2}}{d^{\eta}}
$$

where $E_{\min }(\eta)$ is the energy of the Bravais lattice $\mathcal{L}^{*}(d)$, assuming the minimum energy property of the Bravais lattices in D dimensions. 
Proof: Recall that

$$
U_{o}\left(N^{D}\right)=\sum_{k=1}^{r} U_{w}\left(N_{k}\right)
$$

where $U_{w}\left(N_{k}\right)$ denotes the utility corresponding to the $N_{k}$ nodes in the set $G_{k}$, which are all transmitting in band $b_{k}$. The configuration corresponding to the Bravais lattice with the minimum energy, $\mathcal{L}^{*}\left(\left(\frac{N^{D}}{N_{k}}\right)^{\frac{1}{D}} d\right)$, gives the global minimum of the potential function $V_{G_{k}}$ (the global maximum of $-U_{w}\left(N_{k}\right)$ ). Hence, for any given distribution of the $N^{D}$ nodes inside the $D$ dimensional cube of side $N d$, we have

$$
\lim _{N_{k} \rightarrow \infty} \frac{\frac{1}{N_{k}} U_{w}\left(N_{k}\right)}{\frac{1}{N_{k}} U_{w}\left(N_{k} ; \mathcal{L}^{*}\left(\left(\frac{N^{D}}{N_{k}}\right)^{\frac{1}{D}} d\right)\right)} \leqslant 1
$$

where $U_{w}\left(N_{k} ; \mathcal{L}^{*}\left(\left(\frac{N^{D}}{N_{k}}\right)^{\frac{1}{D}} d\right)\right)$ corresponds to the worst case network utility when the $N_{k}$ nodes are located on the sites of the Bravais lattice $\mathcal{L}^{*}$ with unit spacing $\left(\frac{N^{D}}{N_{k}}\right)^{\frac{1}{D}} d$.

Moreover, it can be shown that $N_{k} \rightarrow \infty$ as $N \rightarrow \infty$ for all $k=1,2, \cdots, r$, where $G_{k} \mathrm{~s}$ correspond to that of the optimal frequency band assignment. This is established by Lemma A.2 in Appendix A. Thus, we have

$$
\begin{aligned}
\lim _{N \rightarrow \infty} \frac{1}{N^{D}} U_{o}\left(N^{D}\right) & =\lim _{N \rightarrow \infty} \frac{1}{N^{D}} \sum_{k=1}^{r} U_{w}\left(N_{k}\right) \\
& \leqslant \lim _{N \rightarrow \infty} \frac{1}{N^{D}} \sum_{k=1}^{r} U_{w}\left(N_{k} ; \mathcal{L}^{*}\left(\left(\frac{N^{D}}{N_{k}}\right)^{\frac{1}{D}} d\right)\right)
\end{aligned}
$$

Also, for an array of nodes located in inside the $D$ dimensional cube of side $N d$ on the sites of the $\mathcal{L}^{*}(d)$ Bravais lattice, it is easy to show that

$$
\lim _{N \rightarrow \infty} \frac{1}{N^{D}} U_{w}\left(N^{D} ; \mathcal{L}^{*}(d)\right)=-E_{\min }(\eta) \frac{P_{0}^{2}}{d^{\eta}}
$$

This result is proven in Lemma A.1 in Appendix A. Roughly speaking, the above equation states that the boundary effects of the lattice can be neglected in the limit of $N \rightarrow \infty$. Using the foregoing result, the right-hand side of Eq. (45) can be bounded as follows:

$$
\lim _{N \rightarrow \infty} \frac{1}{N^{D}} \sum_{k=1}^{r} U_{w}\left(N_{k} ; \mathcal{L}^{*}\left(\left(\frac{N^{D}}{N_{k}}\right)^{\frac{1}{D}} d\right)\right) \leqslant \lim _{N \rightarrow \infty}\left(\frac{\sum_{k=1}^{r} N_{k}^{\frac{\eta}{D}+1}}{N^{\eta+D}}\right) \frac{1}{N^{D}} U_{w}\left(N^{D} ; \mathcal{L}^{*}(d)\right)
$$


The expression $\sum_{k=1}^{r} N_{k}^{\frac{\eta}{D}+1}$ clearly achieves its minimum when $N_{k}=N^{D} / r$, for $k=$ $1, \cdots, r$. Therefore,

$$
\begin{aligned}
\lim _{N \rightarrow \infty} \frac{1}{N^{D}} U_{o}\left(N^{D}\right) & \leqslant \lim _{N \rightarrow \infty}\left(\frac{\sum_{k=1}^{r} N_{k}^{\frac{\eta}{D}+1}}{N^{\eta+D}}\right) \frac{1}{N^{D}} U_{w}\left(N^{D} ; \mathcal{L}^{*}(d)\right) \\
& \leqslant \frac{1}{r^{\frac{\eta}{D}}} \lim _{N \rightarrow \infty} \frac{1}{N^{D}} U_{w}\left(N^{D} ; \mathcal{L}^{*}(d)\right) \\
& =-\frac{1}{r^{\frac{\eta}{D}}} E_{\min }(\eta) \frac{P_{0}^{2}}{d^{\eta}}
\end{aligned}
$$

which proves the statement of Theorem 4.3.

Remark: Note that the network utility $U\left(N^{D}\right)$ is an extensive variable, i.e., it scales with the number of nodes $N^{D}$. The normalization factor $1 / N^{D}$ in the result of Theorem 4.3 (and similar theorems that follow) guarantees that the limits are well-defined.

We can prove the following stronger result for the alternating frequency band assignment to the nodes located on the Bravais lattice with minimum energy:

Theorem 4.4: Let $U_{\text {alt }}\left(N^{D} ; \mathcal{L}^{*}(d)\right)$ be the network utility corresponding to the alternating frequency band assignment to the nodes located on the $D$ dimensional Bravais lattice $\mathcal{L}^{*}(d)$ with minimum energy $E_{\min }(\eta)$ and unit spacing $d$. Also, suppose that $P_{i}=P_{0}$ for all $1 \leqslant i \leqslant N$. Then, we have

$$
\lim _{N \rightarrow \infty} \frac{1}{N^{D}} U_{\text {alt }}\left(N^{D} ; \mathcal{L}^{*}(d)\right)=-\frac{1}{r^{\frac{\eta}{D}}} E_{\min }(\eta) \frac{P_{0}^{2}}{d^{\eta}}
$$

Proof: Suppose that we alternatingly assign frequency bands $b_{1}, b_{2}, \cdots, b_{r}$ to the nodes located on the $r$ cosets of $\mathcal{L}^{*}(d)$. First, we will show that

$$
r U_{w}\left(\left\lfloor N^{D} / r\right\rfloor ; \mathcal{L}^{*}\left(r^{1 / D} d\right)\right) \geqslant U_{\text {alt }}\left(N^{D} ; \mathcal{L}^{*}(d)\right) \geqslant r U_{w}\left(\left\lceil N^{D} / r\right\rceil ; \mathcal{L}^{*}\left(r^{1 / D} d\right)\right)
$$

To see this, we note that in the alternating frequency band assignment there are $\left\lfloor N^{D} / r\right\rfloor \leqslant$ $N_{k} \leqslant\left\lceil N^{D} / r\right\rceil$ nodes located on the coset $\mathcal{L}^{*}\left(r^{1 / D} d\right)$ which are all operating in frequency band $b_{k}$, for $k=1,2, \cdots, r$. Therefore, the network utility is given by the sum of network utilities of the $r$ disjoint sets of nodes corresponding to different frequency bands. Clearly, all the nodes 
in each subset have the same frequency band and therefore, the network utility for subset $G_{k}$, with $\left|G_{k}\right|=N_{k}$, is $U_{w}\left(N_{k} ; \mathcal{L}^{*}\left(r^{1 / D} d\right)\right)$. We can write $U_{\text {alt }}(N ; d)$ as follows:

$$
U_{\text {alt }}\left(N^{D} ; \mathcal{L}^{*}(d)\right)=\sum_{k=1}^{r} U_{w}\left(N_{k} ; \mathcal{L}^{*}\left(r^{1 / D} d\right)\right)
$$

Since $U_{w}(N ; \cdot)$ is an increasing function of $N$, we can sandwich $U_{w}\left(N_{k} ; \mathcal{L}^{*}\left(r^{1 / D} d\right)\right)$ as

$$
U_{w}\left(\left\lfloor N^{D} / r\right\rfloor ; \mathcal{L}^{*}\left(r^{1 / D} d\right)\right) \geqslant U_{w}\left(N_{k} ; \mathcal{L}^{*}\left(r^{1 / D} d\right)\right) \geqslant U_{w}\left(\left\lceil N^{D} / r\right\rceil ; \mathcal{L}^{*}\left(r^{1 / D} d\right)\right)
$$

Summing over $k$ yields

$$
r U_{w}\left(\left\lfloor N^{D} / r\right\rfloor ; \mathcal{L}^{*}\left(r^{1 / D} d\right)\right) \geqslant U_{\text {alt }}\left(N^{D} ; \mathcal{L}^{*}(d)\right) \geqslant r U_{w}\left(\left\lceil N^{D} / r\right\rceil ; \mathcal{L}^{*}\left(r^{1 / D} d\right)\right)
$$

where we have used Eq. (51). Using the Sandwich Theorem, we get

$$
\begin{aligned}
\lim _{N \rightarrow \infty} \frac{1}{N^{D}} U_{\text {alt }}\left(N^{D} ; \mathcal{L}^{*}(d)\right) & =\lim _{N \rightarrow \infty} \frac{1}{N^{D}} r U_{w}\left(\left\lceil N^{D} / r\right\rceil ; \mathcal{L}^{*}\left(r^{1 / D} d\right)\right) \\
& =\lim _{N \rightarrow \infty}-\frac{\left\lceil N^{D} / r\right\rceil}{N^{D} / r} E_{\min }(\eta) \frac{P_{0}^{2}}{\left(r^{1 / D} d\right)^{\eta}} \\
& =-\frac{1}{r^{\frac{\eta}{D}}} E_{\min }(\eta) \frac{P_{0}^{2}}{d^{\eta}}
\end{aligned}
$$

On the other hand, from Theorem 4.3 we know that

$$
\lim _{N \rightarrow \infty} \frac{1}{N^{D}} U_{o}\left(N^{D}\right) \leqslant-\frac{1}{r^{\frac{\eta}{D}}} E_{\min }(\eta) \frac{P_{0}^{2}}{d^{\eta}}
$$

Therefore,

$$
\lim _{N \rightarrow \infty} \frac{1}{N^{D}} U_{o}\left(N^{D} ; \mathcal{L}^{*}(d)\right)=\lim _{N \rightarrow \infty} \frac{1}{N^{D}} U_{\text {alt }}\left(N^{D} ; \mathcal{L}^{*}(d)\right)=-\frac{1}{r^{\frac{\eta}{D}}} E_{\min }(\eta) \frac{P_{0}^{2}}{d^{\eta}}
$$

which proves the statement of the theorem.

Combining the results of Theorems 4.2 and 4.3, the following theorem compares the performance of the GADIA algorithm to that of the optimal strategy:

Theorem 4.5: Consider a given spatial distribution of $N^{D}$ nodes inside the $D$ dimensional cube with side $N d$. Suppose that $P_{i}=P_{0}$ for all $1 \leqslant i \leqslant N$. Moreover, let $U_{G}\left(N^{D}\right)$ be the network utility corresponding to the output of the GADIA algorithm and $U_{o}\left(N^{D}\right)$ be that of the optimal frequency band assignment. Then, we have

$$
\gamma_{D} \lim _{N \rightarrow \infty} \frac{1}{N^{D}} U_{o}\left(N^{D}\right) \leqslant \lim _{N \rightarrow \infty} \frac{1}{N^{D}} U_{G}\left(N^{D}\right) \leqslant \lim _{N \rightarrow \infty} \frac{1}{N^{D}} U_{o}\left(N^{D}\right),
$$


where

$$
\gamma_{D}:=r^{\frac{\eta}{D}-1} \frac{E_{\max }(\eta)}{E_{\min }(\eta)} \frac{1}{\left(\frac{d_{\min }}{d}\right)^{\eta}},
$$

$d_{\min }:=\min _{i, j} d_{i j}$, and $E_{\min }(\eta)$ and $E_{\max }(\eta)$ are the minimum and maximum energies of the Bravais lattices in D dimensions, respectively.

Proof: Clearly, we have

$$
U_{w}\left(N^{D}\right) \geqslant U_{w}\left(N^{D} ; \mathcal{L}^{\prime}\left(d_{\text {min }}\right)\right),
$$

where $\mathcal{L}^{\prime}\left(d_{\min }\right)$ is the $D$ dimensional Bravais lattice with the maximum energy, $E_{\max }(\eta)$, and spacing $d_{\min }$. In other words, for any given spatial configuration of the network nodes with all being in the same frequency band, the worst case utility can be further decreased by locating all the nodes on the sites of the Bravais lattice with the highest energy and unit distance equal to the smallest separation in the original network. Moreover, from Eq. (48) the statement of Theorem 4.3 can be expressed as

$$
\begin{aligned}
\lim _{N \rightarrow \infty} \frac{1}{N^{D}} U_{o}\left(N^{D}\right) & \leqslant \frac{1}{r^{\frac{n}{D}}} \lim _{N \rightarrow \infty} \frac{1}{N^{D}} U_{w}\left(N^{D} ; \mathcal{L}^{*}(d)\right) \\
& =\frac{1}{r^{\frac{n}{D}}}\left(\frac{d_{\min }}{d}\right)^{\eta} \lim _{N \rightarrow \infty} \frac{1}{N^{D}} U_{w}\left(N^{D} ; \mathcal{L}^{*}\left(d_{\min }\right)\right)
\end{aligned}
$$

since $U_{w}\left(N^{D} ; \mathcal{L}^{*}\left(d_{\min }\right)\right)$ depends on $d$ as $1 / d^{\eta}$. Using Theorem 4.2 and the bound given in Eq. (59), we get

$$
\lim _{N \rightarrow \infty} \frac{1}{N^{D}} U_{G}\left(N^{D}\right) \geqslant \frac{1}{r} \lim _{N \rightarrow \infty} \frac{1}{N^{D}} U_{w}\left(N^{D}\right) \geqslant \frac{1}{r} \lim _{N \rightarrow \infty} \frac{1}{N^{D}} U_{w}\left(N^{D} ; \mathcal{L}^{\prime}\left(d_{\min }\right)\right)
$$

Dividing Eq. (61) by Eq. (60) yields:

$$
\frac{\lim _{N \rightarrow \infty} \frac{1}{N^{D}} U_{G}\left(N^{D}\right)}{\lim _{N \rightarrow \infty} \frac{1}{N^{D}} U_{o}\left(N^{D}\right)} \geqslant \frac{1}{r} \cdot \frac{r^{\frac{\eta}{D}}}{\left(\frac{d_{\min }}{d}\right)^{\eta}} \cdot \frac{\lim _{N \rightarrow \infty} \frac{1}{N^{D}} U_{w}\left(N^{D} ; \mathcal{L}^{\prime}\left(d_{\min }\right)\right)}{\lim _{N \rightarrow \infty} \frac{1}{N^{D}} U_{w}\left(N^{D} ; \mathcal{L}^{*}\left(d_{\min }\right)\right)}
$$

Now, Lemma A.1 implies that the ratio of $\frac{1}{N^{D}} U_{w}\left(N^{D} ; \mathcal{L}^{\prime}\left(d_{\min }\right)\right)$ to $\frac{1}{N^{D}} U_{w}\left(N^{D} ; \mathcal{L}^{*}\left(d_{\min }\right)\right)$ is asymptotically given by $E_{\max }(\eta) / E_{\min }(\eta)$. Hence, by rearranging the terms in Eq. (62) we recover

$$
\gamma_{D} \lim _{N \rightarrow \infty} \frac{1}{N^{D}} U_{o}\left(N^{D}\right) \leqslant \lim _{N \rightarrow \infty} \frac{1}{N^{D}} U_{G}\left(N^{D}\right) .
$$

Given the trivial upper bound of $U_{G}\left(N^{D}\right) \leqslant U_{o}\left(N^{D}\right)$, the statement of the Theorem follows. 


\section{Discussion of the Results}

Theorem 4.1 guarantees the convergence of the GADIA algorithm, regardless of the power distribution and spatial configuration of the nodes in the network, as long as the hypothesis of interference reciprocity holds. Theorem 4.2 establishes a lower bound on the network utility corresponding to the GADIA algorithm, which holds for any spatial distribution of the network nodes. However, this bound also requires the hypothesis of interference symmetry. It is worth mentioning that the symmetric interference requirement can be restrictive in generalizing the application of the GADIA algorithm to other network models. For example, if the network nodes are equipped with multiple transmitters and receivers, the assumption of symmetric interference does not hold in general [45]. Moreover, realization of channel reciprocity might exhibit difficulties in practice (See [18] for a detailed discussion). However, as far as the network model in this paper and those studied in [34], [35] and [36] are concerned, this requirement can be enforced with rather mild costs. In particular, Neel [35] has introduced a number of methods to synthetize the symmetric interference condition, which are mainly based on appropriately refining the interference sensing process.

Theorem 4.3 establishes an asymptotic upper bound on the optimal network utility, independent of the spatial distribution of the nodes. Although, in obtaining this bound, properties of the Bravais lattices have been used. Also, note that Theorem 4.3 relies on the widely accepted conjecture that Bravais lattices correspond to the energy minima of particles with repulsive forces. Although this conjecture is supported with numerous experimental results and is widely accepted in solid state physics, it must be noted that the verity of Theorem 4.3 depends on the verity of this conjecture. Theorem 4.4 establishes that the bound obtained in Theorem 4.3 is indeed sharp, i.e., there exists a network with the Bravais structure and a specific frequency band assignment (alternating assignment) which has the same network utility as the bound obtained

in Theorem 4.3. Finally, Theorem 4.5 uses the results of Theorems 4.2 and 4.3 to establish an asymptotic lower bound on the network utility corresponding to the GADIA algorithm, compared to the optimal network utility, for a general spatial distribution of the network nodes. Note that the lower bound of Theorem 4.2 and the asymptotic lower bound of Theorem 4.5 indeed correspond to networks with general spatial distribution of nodes. Simulation results (Section VI) show that these bounds give reasonable estimates of the utility range to which the network utility of the 
GADIA algorithm converges.

The result of Theorem 4.5 is the strongest when the constant $\gamma_{D}$ is not too large. In order to have some numerical intuition about $\gamma_{D}$, we consider some special cases: in 1 dimension, with $\eta=2$ and $r=2$, for a uniform linear array with unit distance $d$, we have $\gamma_{1}=2$, which implies that the output of the GADIA algorithm is guaranteed to be within $3 d B$ of the optimal frequency band assignment. In 2 dimensions the situation is better, since the effect of $r$ on $\gamma_{D}$ is reduced: with $\eta=2.5$ and $r=4$, for a uniform rectangular array with unit distance $d$, we have $\gamma_{2} \approx 1.41$. Hence, the outcome of the GADIA algorithm will be within $1.5 d B$ of the optimal strategy. Note that the result of corollary is a worst-case result which holds for any given spatial configuration of the nodes and any single run of the GADIA algorithm. Simulation results in Section VI indicate that the GADIA algorithm indeed performs within these bounds, and on average performs very close to the optimal strategy.

It is worth mentioning that the lower bound obtained on the performance of the GADIA algorithm can also be interpreted in the framework of Price of Anarchy (PoA) (See, for example [27] and [47]). The notion of PoA denotes the ratio of the aggregate welfare of the optimal Nash equilibrium to the worst case Nash equilibrium of a non-cooperative game with many players. Originally, the PoA has been studied in the context of selfish routing in data networks. In such networks, each node needs to choose a path in a graph to route its message through. Each edge of the graph is weighted according to its latency, which is an increasing function of the number of nodes including it in their routing path. The usual model used for the latency function is the flow model, where the latency is an increasing function of flow (number of users) of that edge. Several results concern linear, polynomial and continuous functions of the flow of each edge as the corresponding latency [47].

In this regard, our network model can be viewed as a collection of $N$ nodes which need to route their message to a destination node, through one of the available $r$ edges (which correspond to the available frequency bands). The latency of each edge is then the interference experienced in the corresponding frequency band. However, the flow model does not necessarily yield a good approximation to the physical properties of our network. For example, two frequency bands may have the same number of users, but the interference experienced by a certain node in each of these bands can be dramatically different due to the spatial configuration of the nodes. Hence, the usual 
results of PoA do not readily generalize to our problem. Moreover, unlike in the flow model, in general it is very hard to characterize the optimal, worst case and other Nash equilibria of our network with the physical interference model. Hence, in the foregoing bounding procedures we have used other techniques to obtain the ratio of the welfare in the Nash equilibrium obtained by the GADIA algorithm to that of the optimal frequency assignment strategy. Nevertheless, the parameter $1 / \gamma_{D}$ can be interpreted as a lower bound on the PoA of the network (since the welfare of the Nash equilibrium obtained by GADIA is greater or equal to that of the worst case Nash equilibrium).

\section{Main Results: Dynamical Analysis}

So far, we have used the discrete-time model in order to carry out the static analysis presented in Section IV. Moreover, most of the results in Section IV correspond to the equilibrium analysis of the algorithm, thus the discrete-time model sufficed to study the algorithm following equilibrium. However, the discrete-time model does not fully capture the response of the algorithm to time variations and stochastic uncertainties. In other words, in order to evaluate the robustness and stability of the algorithm one needs to take into account the continuous-time dynamics.

\section{A. Update Process}

First, we need to model the frequency band update process of the nodes in the network. In general, the update process of each node can be modeled by a stochastic point process. Let $t_{i}^{(1)}, t_{i}^{(2)}, t_{i}^{(3)}, \cdots$ be the points in time when the node $c_{i}$ scans the spectrum in order to update its frequency band. Let $p_{t_{i}^{(\ell+1)}}\left(t ; t_{i}^{(1)}, t_{i}^{(2)}, \cdots, t^{(\ell)}\right)$ be the probability density of $t^{(\ell+1)}$ given the previous points $t_{i}^{(1)}, t_{i}^{(2)}, \cdots, t^{(\ell)}$. The density $p_{t_{i}^{(\ell+1)}}$ is sufficient to describe all the stochastic properties of the point process. In our case, we will model the update process of each node with a Poisson point process of rate $\lambda_{i}[46]$, where

$$
p\left(N_{i}(t+\tau)-N_{i}(t)=k\right)=\frac{e^{-\lambda_{i} \tau}\left(\lambda_{i} \tau\right)^{k}}{k !}
$$

denotes the probability that the node $c_{i}$ updates its frequency band a total of $k$ times in the interval $(t, t+\tau]$, for $k=0,1, \cdots$. It is easy to see that [46]

$$
p_{t_{i}^{(\ell+1)}}\left(t ; t_{i}^{(1)}, t_{i}^{(2)}, \cdots, t^{(\ell)}\right)=\lambda_{i} e^{-\lambda_{i}\left(t-t_{i}^{(\ell)}\right)} .
$$


We also assume that different nodes have independent update processes. The assumption of Poisson update processes is mainly made for mathematical simplicity of the dynamical analysis that follows. Similar analysis can be carried out by modeling the update process by other point processes. However, the analytical results will be more complicated (although very similar in essence) and may adumbrate the underlying intuition and application of the results.

\section{B. The Soft GADIA}

Recall that according to the GADIA algorithm, each node, say $c_{i}$, updates its frequency band at time $t^{(\ell)}$ according to the following rule:

$$
s_{i}(t)=\arg \min _{j} I_{c_{i}}^{j}\left(t^{(\ell)}\right)
$$

where $t \in\left(t^{(\ell)}, t^{(\ell+1)}\right]$. This is simply the continuous-time version of the decision criterion given in Eq. (18). We can alternatively consider a probabilistic decision criterion in which the node $c_{i}$ chooses the frequency band $b_{k}$ with probability

$$
p\left(s_{i}(t)=k\right):=\frac{\exp \left(-\beta P_{i} I_{c_{i}}^{k}\left(t^{(\ell)}\right)\right)}{\sum_{j=1}^{r} \exp \left(-\beta P_{i} I_{c_{i}}^{j}\left(t^{(\ell)}\right)\right)}
$$

for $k=1,2, \cdots, r$ and $t \in\left(t^{(\ell)}, t^{(\ell+1)}\right]$, where $\beta$ is a positive constant. Note that in the limit of $\beta \rightarrow \infty$, the probabilistic decision criterion coincides with that of the GADIA algorithm, since

$$
p\left(s_{i}(t)=k^{*}\right)=1
$$

where $k^{*}:=\arg \min _{k} I_{c_{i}}^{k}\left(t_{i}^{(\ell)}\right)$, and $p\left(s_{i}(t)=k\right)=0$ for all $k \neq k^{*}$, as $\beta \rightarrow \infty$. We note that $\beta$ is a measure of deviation from the optimal decision by the nodes in the network. Therefore, in analogy to statistical physics conventions, we denote by $\beta$ the inverse temperature. We also denote by Soft GADIA the frequency allocation algorithm with the decision criterion given in Eq. (67). In what follows, we carry out the analysis for the Soft GADIA algorithm due to the smoothness properties of its probability distribution on the state space, and also conforming with the conventions of statistical physics. Nevertheless, the corresponding results for the GADIA algorithm can be deduced from those of Soft GADIA by the limit process $\beta \rightarrow \infty$.

Recall that the state of the network at time $t$ is given by the vector $\left(s_{1}, s_{2}, \cdots, s_{N}\right) \in$ $\{1,2, \cdots, r\}^{N}$ corresponding to the frequency bands occupied by nodes $\left(c_{1}, c_{2}, \cdots, c_{N}\right)$. Let 
$P\left(t ; s_{1}, s_{2}, \cdots, s_{N}\right)$ be the probability density of the nodes $\left(c_{1}, c_{2}, \cdots, c_{N}\right)$ being at the point $\left(s_{1}, s_{2}, \cdots, s_{N}\right)$ in the state-space at time $t$. For any scalar function $f\left(s_{1}, s_{2}, \cdots, s_{N}\right)$ on the state-space, the ensemble average at time $t$ is defined as follows:

$$
\left\langle f\left(s_{1}, s_{2}, \cdots, s_{N}\right)\right\rangle:=\sum_{\sigma_{1}=1}^{r} \sum_{\sigma_{2}=1}^{r} \cdots \sum_{\sigma_{N}=1}^{r} f\left(\sigma_{1}, \sigma_{2}, \cdots, \sigma_{N}\right) P\left(t ; \sigma_{1}, \sigma_{2}, \cdots, \sigma_{N}\right)
$$

In particular, we will prove the following theorem regarding the dynamics of the Soft GADIA algorithm:

Theorem 5.1: Let $U(t)$ be the network utility at time $t$, corresponding to the Soft GADIA algorithm with parameter $\beta$. Moreover, suppose that the network nodes update their frequency bands with a rate $\lambda$, i.e., $\lambda_{i}=\lambda$ for all $i=1,2, \cdots, N$. Then, the ensemble average of the network utility satisfies the following differential equation:

$$
\frac{d}{d t}\langle U(t)\rangle=-2 \lambda\left(\langle U(t)\rangle-\left\langle U_{0}(t)\right\rangle\right)
$$

where

$$
U_{0}(t):=-\sum_{\substack{k, l=1 \\ k \neq l}}^{N} P_{k} P_{l} \alpha_{k l} \frac{\exp \left(-\beta \sum_{j \neq k} P_{k} P_{j} \alpha_{k j} \delta\left(s_{l}(t), s_{j}(t)\right)\right)}{\sum_{m} \exp \left(-\beta \sum_{j \neq k} P_{k} P_{j} \alpha_{k j} \delta\left(m, s_{j}(t)\right)\right)} .
$$

Before proving Theorem 5.1, we need to introduce some concepts from statistical physics, which are key to analyzing the dynamical performance of the Soft GADIA algorithm and, in particular, proving Theorem 5.1.

\section{Connection to Potts Model}

In the continuous-time model, the network utility is given by

$$
U(t)=-\sum_{i=1}^{N} P_{i} I_{c_{i}}(t)=-\sum_{\substack{i, j=1 \\ i \neq j}}^{N} P_{i} P_{j} \alpha_{i j} \delta\left(s_{i}(t), s_{j}(t)\right)
$$

where we have approximated the leakage function $f\left(s_{i}, s_{j}\right)$ by the Kronecker delta function $\delta\left(s_{i}, s_{j}\right)$. Let $J_{i j}:=P_{i} P_{j} \alpha_{i j}$ for $1 \leqslant i, j \leqslant N, i \neq j$. Clearly, $J_{i j}=J_{j i}$, by the hypothesis of interference reciprocity $\left(\alpha_{i j}=\alpha_{j i}\right)$. The network utility can be expressed as

$$
U(t)=-\sum_{\substack{i, j=1 \\ i \neq j}}^{N} J_{i j} \delta\left(s_{i}(t), s_{j}(t)\right)
$$


The above expression can be identified with the Hamiltonian of an anti-ferromagnetic inhomogeneous r-state long-range Potts model [55]. The Potts model is a generalization of the Ising model, which describes the interactions of spins on a crystalline lattice, and is studied extensively in the context of solid state physics (See, for example, [8], [24] and [55]).

In the original Potts model, the summation is only over the nearest neighbors and is thus denoted by short-range Potts model. Moreover, $J_{i j}=-J$ for all $i$ and $j$. Therefore, the network utility given in Eq. (71) corresponds to the inhomogeneous long-range generalization of the original Potts model. Also, note that any two nearby nodes tend to be in different frequency bands. Hence, the model is anti-ferromagnetic in nature, which is reflected in the negativeness of the network utility. Another generalization, called the infinite-range Potts model, has been widely studied in statistical physics, which corresponds to the case where $J_{i j}=1 / N$ for all $i, j$ and the summation is over all the spins on the lattice (not only the nearest neighbors) [55]. Although the energetics of the infinite-range Potts model is analytically tractable, its generalization to the inhomogeneous long-range case is not trivial.

\section{Glauber Dynamics}

In light of the above, we can analyze the temporal dynamics of the soft GADIA algorithm in the context of spin dynamics. Study of spin dynamics was initiated by the seminal paper of Roy J. Glauber [19] and is thus commonly denoted by Glauber dynamics. The Glauber dynamics was originally devised in order to describe the near equilibrium collective behavior of the spins on a lattice, interacting according to the one-dimensional Ising model [19]. Generalizations to other spin models such as the original Potts model and infinite-range Potts model have been done (See, for example, [6] and [29]). The decision criterion given in Eq. (67) is a generalization of the Glauber dynamics to the anti-ferromagnetic inhomogeneous $r$-state long-range Potts model.

Let

$$
w_{i}(s):=p\left(s_{i}(t)=s\right)
$$

for all $s \in\{1,2, \cdots, r\}$, which is given by Eq. (67). We note that $w_{i}(s)$ is implicitly a function of $t$, but we drop the dependence on $t$ for notational convenience. Recall that $P\left(t ; s_{1}, s_{2}, \cdots, s_{N}\right)$ is the probability density of the nodes $\left(c_{1}, c_{2}, \cdots, c_{N}\right)$ being at the point $\left(s_{1}, s_{2}, \cdots, s_{N}\right)$ in the state-space at time $t$. As Glauber noted in his paper [19], the stochastic properties of such 
systems (and variants thereof) can be fully described by the Master equation [32]:

$$
\begin{aligned}
\frac{\partial}{\partial t} P\left(t ; s_{1}, s_{2}, \cdots, s_{N}\right) & =\sum_{i=1}^{N} \lambda_{i} w_{i}\left(s_{i}\right) \sum_{\substack{\sigma_{j}=1 \\
\sigma_{j} \neq s_{j}}}^{r} P\left(t ; s_{1}, s_{2}, \cdots, \sigma_{j}, \cdots, s_{N}\right) \\
& -\sum_{i=1}^{N} \sum_{\substack{\sigma_{j}=1 \\
\sigma_{j} \neq s_{j}}}^{r} \lambda_{i} w_{i}\left(\sigma_{j}\right) P\left(t ; s_{1}, s_{2}, \cdots, s_{N}\right) .
\end{aligned}
$$

for any configuration $\left(s_{1}, s_{2}, \cdots, s_{N}\right)$. The first term on the right-hand side of the Master equation corresponds to the probability flow of the nodes switching to the configuration given by $\left(s_{1}, s_{2}, \cdots, s_{N}\right)$ from any other configuration and thus appears with a positive sign. The second terms corresponds to the probability flow of the nodes which are currently in states $\left(s_{1}, s_{2}, \cdots, s_{N}\right)$ and are switching to other configurations, which appears with a negative sign. Note that the state-space has $r^{N}$ distinct points of the form $\left(s_{1}, s_{2}, \cdots, s_{N}\right)$. Thus, the Master equation is a set of $r^{N}$ coupled equations for the density functions $P\left(t ; s_{1}, s_{2}, \cdots, s_{N}\right)$. Although simultaneously solving this set of equations is mathematically intractable, the Master equation is very useful for computing the time evolution of the statistical parameters of the network.

Proof of Theorem 5.1: We need to compute the ensemble average of the network utility, $\langle U(t)\rangle$, which represents the expected value of the utility function at time $t$. In order to do this, we need to compute ensemble averages of the form $\left\langle\delta\left(s_{k}(t), s_{l}(t)\right)\right\rangle$ for all $1 \leqslant k, l \leqslant N$. As it is shown in Appendix B, the time evolution of $\left\langle\delta\left(s_{k}(t), s_{l}(t)\right)\right\rangle$ is given by the following differential equation:

$$
\begin{aligned}
\frac{d}{d t}\left\langle\delta\left(s_{k}(t), s_{l}(t)\right)\right\rangle & =-\left(\lambda_{k}+\lambda_{l}\right)\left\langle\delta\left(s_{k}(t), s_{l}(t)\right)\right\rangle \\
& +\left\langle\lambda_{k} \frac{\exp \left(-\beta \sum_{j \neq k} J_{k j} \delta\left(s_{l}(t), s_{j}(t)\right)\right)}{\sum_{m} \exp \left(-\beta \sum_{j \neq k} J_{k j} \delta\left(m, s_{j}(t)\right)\right)}\right\rangle \\
& +\left\langle\lambda_{l} \frac{\exp \left(-\beta \sum_{j \neq l} J_{l j} \delta\left(s_{k}(t), s_{j}(t)\right)\right)}{\sum_{m} \exp \left(-\beta \sum_{j \neq l} J_{l j} \delta\left(m, s_{j}(t)\right)\right)}\right\rangle
\end{aligned}
$$

for all $1 \leqslant k, l \leqslant N$. Although the above set of $N(N-1) / 2$ non-linearly coupled differential equations are very hard to solve, they are sufficient to describe the time evolution of $\langle U(t)\rangle$. 
The ensemble average of $U(t)$ can be written as

$$
\langle U(t)\rangle=-\sum_{k, l=1, k \neq l}^{N} J_{k l}\left\langle\delta\left(s_{k}(t), s_{l}(t)\right)\right\rangle .
$$

Given that $\lambda_{i}=\lambda$ for all $i=1,2, \cdots, N$, combining Eqs. (74) and (75) yields:

$$
\frac{d}{d t}\langle U(t)\rangle=-2 \lambda\left(\langle U(t)\rangle-\left\langle U_{0}(t)\right\rangle\right)
$$

where

$$
U_{0}(t):=-\sum_{\substack{k, l=1 \\ k \neq l}}^{N} J_{k l} \frac{\exp \left(-\beta \sum_{j \neq k} J_{k j} \delta\left(s_{l}(t), s_{j}(t)\right)\right)}{\sum_{m} \exp \left(-\beta \sum_{j \neq k} J_{k j} \delta\left(m, s_{j}(t)\right)\right)} .
$$

Noting that $J_{i j}=P_{i} P_{j} \alpha_{i j}$, the statement of the theorem follows.

Eq. (76) (together with the set of equations given by Eq. (74)) gives the complete description of the collective behavior of the network under the soft GADIA algorithm, at all times.

\section{E. Near Equilibrium Linearization}

Note that Eq. (76) has been derived for the utility model $U(t)$ in its most general form (with the exception of approximating the leakage function by the Kronecker delta function). In other words, for any interference reciprocal model, any spatial distribution of nodes and any number of available frequency bands, the behavior of the ensemble average of the utility function is governed by Eq. (76).

However, it is possible to further simplify Eq. (76) near the equilibrium, with appropriate assumptions. First we note that:

$$
\lim _{t \rightarrow \infty}\langle U(t)\rangle=\lim _{t \rightarrow \infty}\left\langle U_{0}(t)\right\rangle
$$

Let $\langle U(\infty)\rangle:=\lim _{t \rightarrow \infty}\langle U(t)\rangle$. Furthermore, we need to characterize the behavior of the function $U_{0}(t)$ near equilibrium. In the mean-field theory [24], we get:

$$
\left\langle U_{0}(t)\right\rangle \approx-\sum_{k, l=1, k \neq l}^{N} J_{k l} \epsilon_{k l}(t)
$$

where

$$
\epsilon_{k l}(t):=\frac{\exp \left(-\beta \sum_{j \neq k} J_{k j}\left\langle\delta\left(s_{l}(t), s_{j}(t)\right)\right\rangle\right)}{\sum_{m} \exp \left(-\beta \sum_{j \neq k} J_{k j}\left\langle\delta\left(m, s_{j}(t)\right)\right\rangle\right)} .
$$


Clearly, $0 \leqslant \epsilon_{k l}(t) \leqslant 1$, for all $k, l$ and $t$. Let

$$
\xi(t):=\min _{k} \min _{\substack{m, n \\ m \neq n}}\left|\sum_{j \neq k} J_{k j}\left(\left\langle\delta\left(m, s_{j}(t)\right)\right\rangle-\left\langle\delta\left(n, s_{j}(t)\right)\right\rangle\right)\right|_{+}
$$

where $x_{+}:=\max (x, 0)$ for all $x \in \mathbb{R}$. Note that $\xi(t)>0$ for all $t$. For $\beta \gg 1$, any change in the values of $\left\langle\delta\left(s_{k}(t), s_{l}(t)\right)\right\rangle$ less than $\xi(t) / 2 N$, will not change the value of $\epsilon_{k l}(t)$. To see this, note that in the limit $\beta \rightarrow \infty$, for a generic distribution of the coefficients $\left\{J_{i j}\right\}$ and for a fixed user $c_{k}, \epsilon_{k l}(t)=0$ for all $l \neq l^{*}$, and $\epsilon_{k l^{*}}(t)=1$, i.e., $s_{l^{*}}(t)$ is the frequency band in which user $c_{k}$ experiences the least amount of interference (It is possible that $\epsilon_{k l}(t) \neq 0$ for more than one $l$ for a given distribution of $\left\{J_{i j}\right\}$, i.e., there are two frequency bands in which user $c_{k}$ experiences the same level of interference. But, the Lebesgue measure of such distributions is zero among all possible distributions of $\left\{J_{i j}\right\}$ for large enough $N$, since such distributions need to satisfy a finite number of linear equations). Thus, $\epsilon_{i j}$ can be written as

$$
\epsilon_{k l}(t):=\frac{\exp \left(-\beta\left(\sum_{j \neq k} J_{k j}\left\langle\delta\left(s_{l}(t), s_{j}(t)\right)\right\rangle-\sum_{j \neq k} J_{k j}\left\langle\delta\left(s_{l^{*}}(t), s_{j}(t)\right)\right\rangle\right)\right)}{1+\sum_{m \neq l^{*}} \exp \left(-\beta\left(\sum_{j \neq k} J_{k j}\left\langle\delta\left(m, s_{j}(t)\right)\right\rangle-\sum_{j \neq k} J_{k j}\left\langle\delta\left(s_{l^{*}}(t), s_{j}(t)\right)\right\rangle\right)\right)} .
$$

Clearly, any change smaller than $\xi(t) / 2 N$ in the exponent of the numerator of the expression in Eq. (82) does not change the limit of $\epsilon_{k l}(t)$ as $\beta \rightarrow \infty$. Hence, for $\langle U(t)\rangle$ sufficiently close to its equilibrium value, $\langle U(\infty)\rangle$, the function $\left\langle U_{0}(t)\right\rangle$ can be considered constant in the mean-field theory. Therefore, for $\beta \gg 1$, we can approximate Eq. (76) near the equilibrium as:

$$
\frac{d}{d t}\langle U(t)\rangle=-2 \lambda(\langle U(t)\rangle-\langle U(\infty)\rangle)
$$

This approximation can be also viewed as near equilibrium linearization. Note that the mean-field theory describes the situation where there are a large number of interferers for each user, i.e., when the network is operating in the low SIR regime. Simulation results in Section VI confirm the validity of the mean-field approximation and the near equilibrium linearization. It is worth mentioning that we have previously obtained a similar result for the special case of 2 frequency bands $(r=2)$ with more elementary arguments [2], [4]. However, the treatment here is much more general and rigorous. 


\section{F. Robustness with respect to Time-varying Node Activities}

As an application of the near equilibrium dynamics of the GADIA algorithm, we want to evaluate the robustness of the algorithm with respect to time-varying activity of the network nodes. That is, we want to generalize the network structure to the case where the nodes can be in active or sleep mode. Let $a_{i}(t)$ be the activity state of the node $c_{i}$ at time $t$. When the node is active at time $t$, i.e., is transmitting, we have $a_{i}(t)=1$ and when the node is in sleep mode, i.e., is not transmitting, we have $a_{i}(t)=0$. A simple stochastic model for the activity of the node $c_{i}$ is the two-state symmetric Markov model with transition probability $\mu_{i}$. This model can be represented in the Itô form as follows (See, for example, [17] and [25]):

$$
d a_{i}(t)=\left(1-a_{i}(t)\right) d N_{i}
$$

where $d N_{i}$ is a Poisson counter with rate $\mu_{i}$. Note that $d N_{i}$ represents a Poisson jump process with rate $\mu_{i}$, such that

$$
\mathcal{E}\left\{d N_{i}\right\}=\mu_{i} d t
$$

where $\mathcal{E}$ denotes the averaging operator corresponding to the Poisson jump process [17]. For simplicity, suppose that $\mu_{i}=\mu$ for all $i$, i.e., all the nodes have the same temporal activity statistics. Also, let $P_{i}=P_{0}$ for all $c_{i}$. The following theorem establishes the steady state behavior of the GADIA algorithm under time-varying node activities:

Theorem 5.2: Let $U(t)$ be the network utility corresponding to the GADIA algorithm at time t. Suppose that $\lambda_{i}=\lambda, \mu_{i}=\mu$ and $P_{i}=P_{0}$ for all $i=1,2, \cdots, N$. Then, the normalized steady state variance of the network utility is given by:

$$
\sigma_{s s}^{2}:=\frac{\lim _{t \rightarrow \infty} \mathcal{E}\left\{(\langle U(t)\rangle-\mathcal{E}\{\langle U(t)\rangle\})^{2}\right\}}{\lim _{t \rightarrow \infty} \mathcal{E}\left\{\langle U(t)\rangle^{2}\right\}}=\frac{4 \mu}{\lambda N-4 \mu}
$$

where $\mathcal{E}\{\cdot\}$ denotes the expectation with respect to the time-varying activity statistics.

Proof: First, note that in the mean-field approximation, every node which switches from the sleep mode to the active mode at time $t$ experiences an interference of $\langle U(t)\rangle / P_{0} N_{a}(t)$, where $N_{a}(t)$ is the number of active nodes at time $t$. Hence, it will decrease the network utility by a total of $2\langle U(t)\rangle / N_{a}(t)$ (Similarly, any node which switches to the sleep mode increases the 
network utility by the same amount). Clearly, we have $N_{a}(t) \approx N / 2$ near equilibrium, since all the nodes have the same temporal activity statistics. Thus, the collective effect of the activity of the nodes can be captured by two Poisson counters in the Itô form as follows:

$$
\frac{2}{N / 2}\langle U(t)\rangle\left(d N_{+}-d N_{-}\right)
$$

where

$$
\mathcal{E}\left\{d N_{ \pm}\right\}=\frac{N}{2} \mu
$$

Hence, the evolution of the network utility near the equilibrium can be described by the following Itô equation:

$$
d\langle U(t)\rangle=-2 \lambda\left(\langle U(t)\rangle-\left\langle U_{0}(t)\right\rangle\right) d t+\frac{4}{N}\langle U(t)\rangle\left(d N_{+}-d N_{-}\right)
$$

We can obtain the Itô equation corresponding to the quantity $\langle U(t)\rangle^{2}$, by the Itô differentiation rule [25] as follows:

$$
\begin{aligned}
d\langle U(t)\rangle^{2} & =-4 \lambda\langle U(t)\rangle\left(\langle U(t)\rangle-\left\langle U_{0}(t)\right\rangle\right) d t \\
& +\left(\left(\langle U(t)\rangle+\frac{4}{N}\langle U(t)\rangle\right)^{2}-\langle U(t)\rangle^{2}\right) d N_{+} \\
& +\left(\left(\langle U(t)\rangle-\frac{4}{N}\langle U(t)\rangle\right)^{2}-\langle U(t)\rangle^{2}\right) d N_{-}
\end{aligned}
$$

Simplifying Eq. (89) and taking the expectation of both sides yields:

$$
\frac{d}{d t} \mathcal{E}\left\{\langle U(t)\rangle^{2}\right\}=-\left(4 \lambda-\frac{16}{N} \mu\right) \mathcal{E}\left\{\langle U(t)\rangle^{2}\right\}+4 \lambda \mathcal{E}\left\{\langle U(t)\rangle\left\langle U_{0}(t)\right\rangle\right\}
$$

Therefore, in the steady state, $t \rightarrow \infty$, we have

$$
\lim _{t \rightarrow \infty} \mathcal{E}\left\{\langle U(t)\rangle^{2}\right\}=\frac{4 \lambda}{4 \lambda-\frac{16}{N} \mu} \mathcal{E}\left\{\langle U(\infty)\rangle^{2}\right\}
$$

given $\lambda>4 \mu / N$. Hence, we have:

$$
\begin{aligned}
\lim _{t \rightarrow \infty} \mathcal{E}\left\{(\langle U(t)\rangle-\mathcal{E}\{\langle U(t)\rangle\})^{2}\right\} & =\frac{4 \lambda}{4 \lambda-\frac{16}{N} \mu} \mathcal{E}\left\{\langle U(\infty)\rangle^{2}\right\}-\mathcal{E}\left\{\langle U(\infty)\rangle^{2}\right\} \\
& =\frac{4 \mu}{\lambda N-4 \mu} \mathcal{E}\left\{\langle U(\infty)\rangle^{2}\right\}
\end{aligned}
$$

which proves the statement of the theorem.

Simulation results in Section VI show that Theorem 5.2 gives a reasonable estimate for the steady state variance of the GADIA algorithm under the time-varying activity of the network 
nodes. A similar result has been derived in [4] for the special case of $r=2$, from a different route with more elementary arguments. The robustness of the GADIA algorithm with respect to the spectrum sensing time and the error induces by simultaneous spectrum sensing by different nodes is studied in [2].

\section{G. Discussion}

Section V-A models the asynchronous update process as a Poisson process. The Poisson process reflects the homogeneity of the network with respect to the update procedure. A more general version of the GADIA algorithm is introduced in Section V-B, denoted by the Soft GADIA algorithm. The difference of the GADIA algorithm with the Soft GADIA is in the decision criterion, which is probabilistic in the latter. The probability of choosing a frequency band is given by the Boltzmann distribition with inverse temperature $\beta$. In the limit of $\beta \rightarrow \infty$, the Boltzmann distribution assigns probability 1 to choosing the frequency band with the least amount of interference. This limiting case clearly coincides with the decision criterion of the GADIA algorithm. However, as mentioned earlier, it is more convenient to analyze the dynamics of the Soft GADIA algorithm. The corresponding dynamics of the GADIA algorithm can be obtained by carrying out the limit of $\beta \rightarrow \infty$. Finally, Theorem 5.1 establishes the dynamical behavior of the ensemble average of the network utility corresponding to the Soft GADIA algorithm.

Section V-C delineates the connection between the negated aggregate interference metric and the Hamiltonian of an anti-ferromagnetic inhomogeneous long-range Potts model. With this connection in mind, one can view the Soft GADIA algorithm as the Glauber dynamics generalized to the anti-ferromagnetic inhomogeneous long-range Potts model. Using the properties of the Glauber dynamics (which is well-studied in statistical physics), Theorem 5.1 regarding the ensemble averaged behavior of the Soft GADIA (and consequently the GADIA) algorithm has been proved in Section V-D. Section V-E presents the near-equilibrium linearization of the dynamics. In fact, it is shown that in the limit of $\beta \rightarrow \infty$, the dynamics can be simplified to a linear first order differential equation with a constant inhomogeneous term (See Eq. (83)). The linearized dynamics can be then used to study the dynamical behavior of the algorithm near the equilibrium. 
As an application, we have studied the robustness of the GADIA algorithm with respect to the time variations in the node activities in Section V-F. In particular, Theorem 5.2 establishes the steady state variance of the network utility corresponding to the GADIA algorithm under the mentioned time-varying setup and in the mean-field approximation. The proof uses modeling techniques and mathematical tools from stochastic control theory and Itô calculus. In fact, the stochastic differential equation given in Eq. (88) gives the full statistical description of the near-equilibrium behavior of GADIA under time-varying node activities. The first term on the right-hand side of Eq. (88) corresponds to the negative drift of the GADIA algorithm which is aimed to increase the network utility. The second term corresponds to the fluctuations in the network utility due to time-varying node activities. Eq. (88) can be used to obtain various moments of the network utility. The normalized steady state variance is shown to settle down to a constant as $t \rightarrow \infty$, given $\lambda>4 \mu / N$. The latter condition implies that the nodes need to update their frequency band roughly $4 / N$ times faster than their on/off switching rate, in order to enjoy a finite variance in the steady-state. Hence, the analysis reveals that as the rate of on/off switching increases, the update rate needs to increase proportionally in order to maintain stability.

\section{Simulation Results}

First, we compare the performance of the GADIA and Iterative Water-filling algorithms for clustered wireless networks. We consider a rectangular lattice in $D$ dimensions, which spans a $D$ dimensional cube of side $N d$. We then randomly and independently pick the positions of $N^{D}$ cluster-heads around the sites of the lattice according to the uniform distribution $[-d / 4, d / 4]$ in each of the $D$ directions. Also, we assume that each cluster-head is transmitting its signal to a user located at a distance $d$ apart, which is also roughly the average distance to the nearest interferers. Fig. 4 shows the performance of the GADIA and Iterative Water-filling algorithms for different configurations of 100 nodes in one and two dimensions. Fig. 4 (a), (b) and (c) show the ensemble-averaged normalized sum-rate of the network as a function of time in one dimension with $r=2, r=4$ and in 2 dimensions with $r=4$, respectively. The normalized sum-rate is defined as the sum-rate divided by the number of clusters. Here $d=1, P_{0}=1$ and $\eta=2$. In two dimensions, the computation of the optimal frequency band assignment is very complicated and finding it by exhaustive search is beyond the capabilities of our simulation platforms. However, 
since the deviation from the rectangular geometry is relatively small, we have instead compared the performance of the GADIA and Iterative Water-filling algorithms to that of the $1: 4$ frequency reuse pattern as a near-optimal candidate [44]. Note that the $1: 4$ frequency reuse pattern, which is used in cellular telephony, coincides with the alternating frequency band assignment introduced in Section IV-C. As it can be observed from Fig. 4, in all cases more than $90 \%$ of the capacity of the optimal (near-optimal) centralized frequency assignment is achieved. Moreover, the GADIA algorithm exhibits a faster convergence rate compared to the Iterative Water-filling algorithm. The results also clearly justify the connection between the weighted aggregate interference and sum-rate.
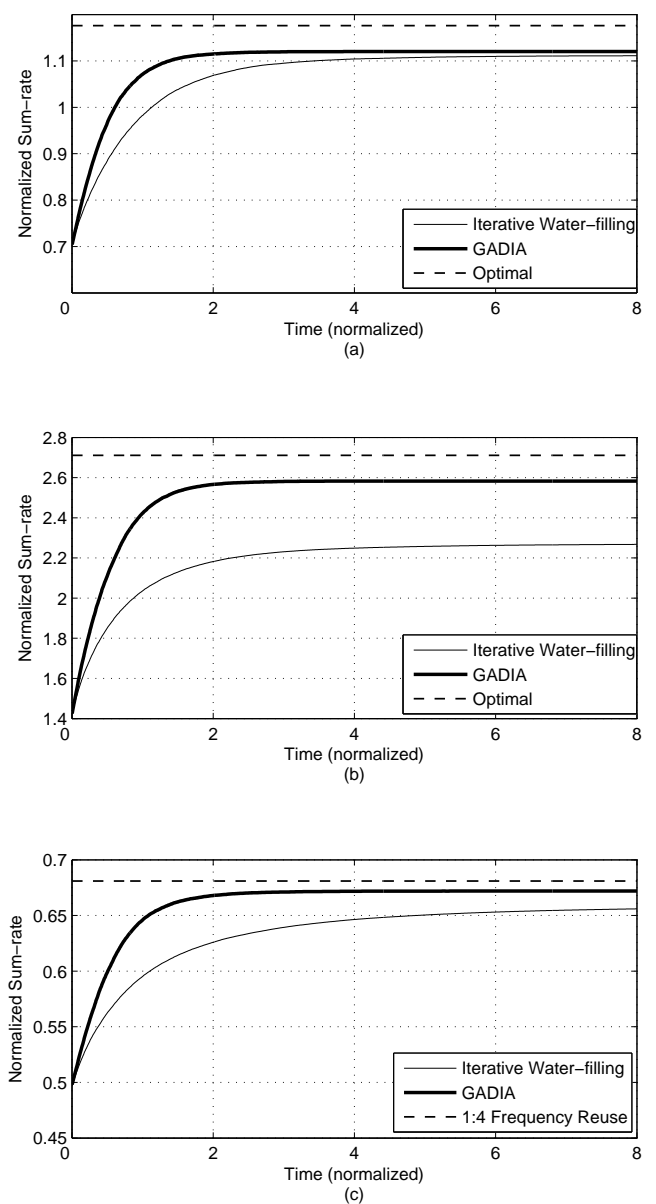

Fig. 4. Normalized sum-rate curves for arrays of 100 clusters vs. time, (a) $1 \mathrm{D}, \mathrm{r}=2$, (b) $1 \mathrm{D}, \mathrm{r}=4$, and (c) 2D, r=4

Fig. 5 (a) and (b) show the normalized network utility corresponding to the GADIA algorithm, 

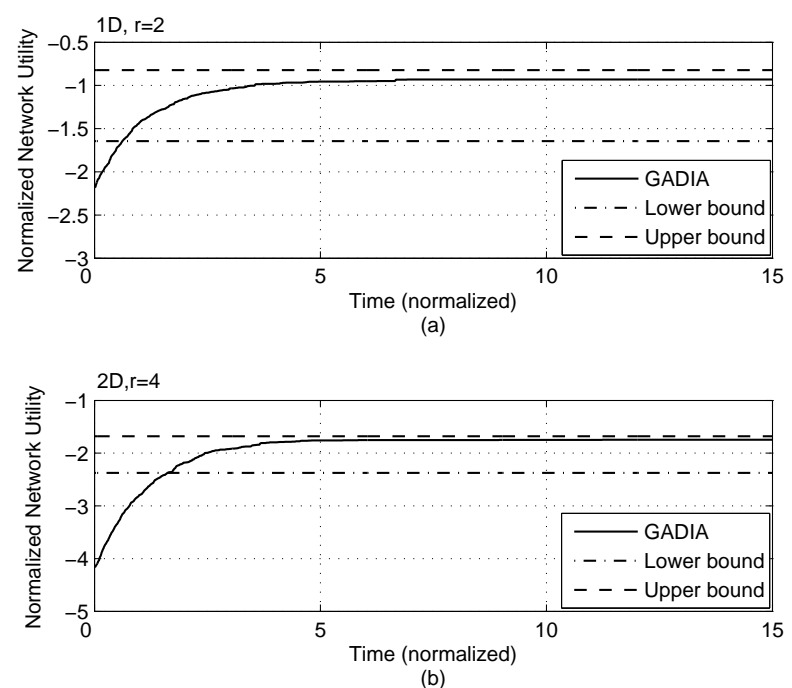

Fig. 5. Normalized network utility of the GADIA algorithm and the theoretical bounds.

the lower and upper bounds we have derived in Section IV-B, for 100 and 400 nodes distributed in one and two dimensions, respectively. Each curve has been obtained by averaging 100 different ensembles. We have set $r=2, \eta=2$ and $r=4, \eta=2.5$ for the one and two dimensional cases, respectively. For the initial condition of the algorithm, we pick a random frequency band for each cluster. The updates are repeated until the convergence is achieved. As we observe from the figure, the GADIA algorithm achieves a network utility very close to the theoretical upper bound.

Fig. 6 shows the average utility of 10 randomly chosen nodes from a rectangular array of 100 nodes vs. time, with $r=4$. The utility is averaged over 50 different runs of the GADIA algorithm on the network. As the GADIA algorithm converges, individual nodes greedily update their frequency band, and eventually remain in the band with the least interference among all.

Fig. 7 shows the performance of the GADIA and Iterative Water-filling algorithms for a linear array of 100 clusters vs. time, in presence of time-varying node activities. For on/off switching probabilities $\mu / N \lambda=0.01,0.05$ and 0.1 , the GADIA algorithm achieves about $90 \%, 86 \%$ and $83 \%$ of the optimal sum-rate on average, respectively. But, Iterative Water-filling achieves about $76 \%, 74 \%$ and $73 \%$ of the optimal sum-rate on average, respectively.

In Fig. 8, the near-equilibrium behavior of the GADIA algorithm for a two dimensional array 


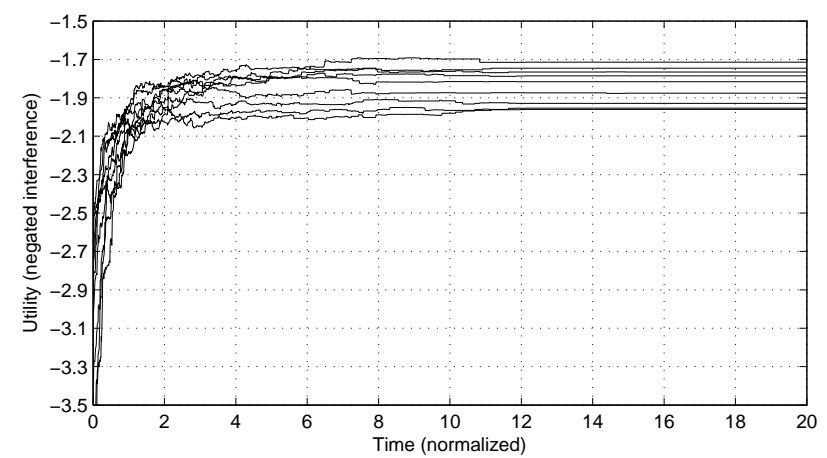

Fig. 6. Average utility of 10 randomly chosen nodes from a rectangular array of 100 nodes vs. time $(r=4)$.
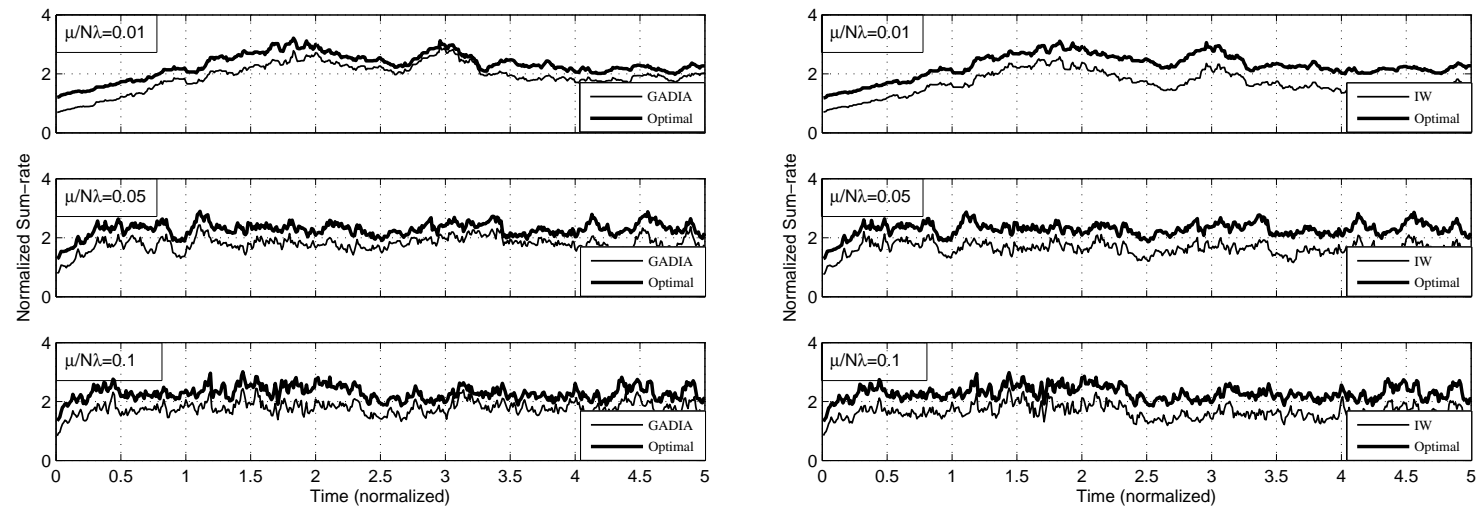

Fig. 7. Normalized sum-rate for a linear array of 100 clusters vs. time, for normalized on/ogg switching probabilities $\mu / N \lambda=$ 0.01, 0.05 and 0.1. Left: GADIA, Right: Iterative Water-filling

of 100 clusters, for $r=2,4$ and 8, is shown. The simulation curve is obtained by averaging over 500 different ensembles. The theoretical estimate of the decay rate of the network utility (which is shown to be $-2 \lambda$ ) matches the simulation data perfectly, which verifies the applicability of the mean field theory to the Glauber dynamics near equilibrium.

Fig. 9 shows the normalized steady state variance of the network utility vs. $\mu / N \lambda$ for a two dimensional array of 100 clusters with $r=4$. The simulation curve is obtained by averaging over 500 different realizations of the update process. As it can be observed from the figure, the theoretical estimate of the steady state variance matches the simulation data perfectly. 

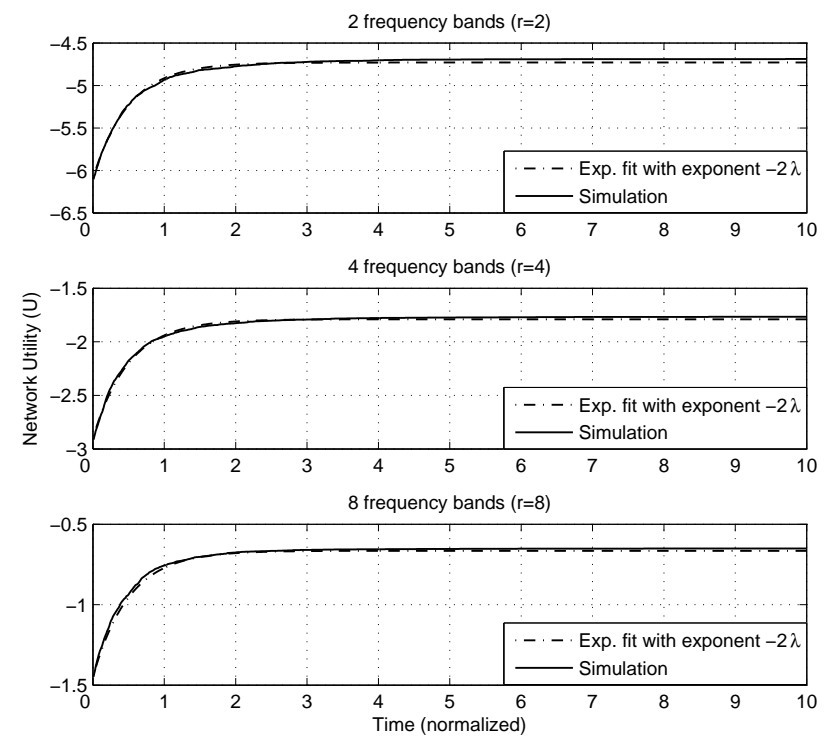

Fig. 8. Normalized network utility vs. time for a 2D array of 100 clusters with $r=2,4$ and 8 .

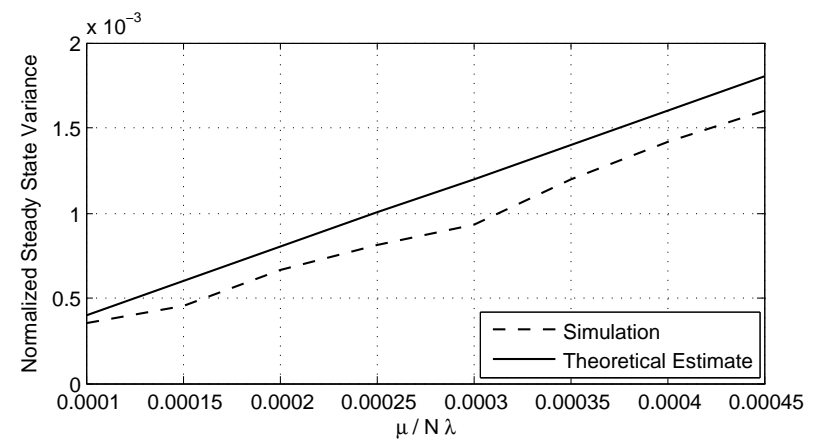

Fig. 9. Normalized steady state variance vs. $\mu / N \lambda$.

\section{CONCLUSION}

We have considered the problem of distributed dynamic frequency allocation in a canonical communication network which spans many networks of interest, such as cognitive/ad hoc networks or Digital Subscriber Lines (DSL). A Greedy Asynchronous Distributed Interference Avoidance (GADIA) algorithm has been proposed that achieves performance close to that of a centralized optimal algorithm. Each node in the network chooses its transmission frequency band based on its knowledge of the interference that it experiences.

The convergence of the proposed distributed algorithm to a near-optimal frequency allocation strategy is proved. Moreover, several asymptotic performance bounds have been derived for 
various spatial configurations of the nodes in the network, by analogy to minimum energy lattice configurations used in solid state physics. These analytic results and additional simulation studies verify performance close to that of an optimum centralized frequency allocation algorithm. In particular, it is demonstrated that the algorithm achieves about $90 \%$ of the sum-rate corresponding to the optimum/near-optimum centralized frequency band assignments. Simulation studies confirm that the GADIA algorithm outperforms the Iterative Water-filling, in the low SIR regime, in terms of the achieved sum-rate, complexity and convergence rate.

Furthermore, the near-equilibrium dynamics of the GADIA algorithm has been studied employing the Glauber dynamics of the anti-ferromagnetic inhomogeneous long-range Potts model. Using the near-equilibrium dynamics and methods from stochastic analysis, the robustness of the algorithm with respect to time variations in the activity of network nodes is studied. It is shown that given a high enough update rate, the network utility enjoys a finite steady-state variance in the presence of time-varying node activities. Further simulation studies confirm the validity of the stochastic modeling and the robustness of the algorithm in the foregoing time-varying setup.

\section{APPENDIX A}

\section{TECHNICAL LEMMAS}

Lemma A.1: Let $U_{w}\left(N^{D} ; \mathcal{L}(d)\right)$ denote the network utility of $N^{D}$ nodes located on the sites of the $\mathcal{L}(d)$ lattice inside the $D$ dimensional cube of side $N d$, when all nodes transmit in the same frequency band. As $N \rightarrow \infty$, we have

$$
\frac{1}{N^{D}} U_{w}\left(N^{D} ; \mathcal{L}(d)\right) \rightarrow-E(\eta) \frac{P_{0}^{2}}{d^{\eta}}
$$

where $E(\eta)$ is the energy of the Bravais lattice $\mathcal{L}(d)$ in $D$ dimensions.

Proof: We present the proof for $D=1$ for brevity. The generalization to $D$ dimensions is straightforward. For $D=1, \mathcal{L}(d)$ corresponds to a uniform linear array on $[0,(N-1) d]$ (i.e., the integer $\mathbb{Z}_{1}$ lattice) [54]. Clearly, we have

$$
\begin{aligned}
\frac{1}{N} U_{w}(N ; d) & =-\frac{1}{N} \frac{P_{0}^{2}}{d^{\eta}} \sum_{i=1}^{N} \sum_{\substack{j=1 \\
j \neq i}}^{N} \frac{1}{|i-j|^{\eta}} \\
& >-\frac{1}{N} \frac{P_{0}^{2}}{d^{\eta}} \sum_{i=1}^{N} 2\left(\sum_{j=1}^{\infty} \frac{1}{j^{\eta}}\right)=-E_{\min }(\eta) \frac{P_{0}^{2}}{d^{\eta}}
\end{aligned}
$$


where

$$
E_{\min }(\eta)=2 \zeta(\eta):=2 \sum_{i=1}^{\infty} \frac{1}{i^{\eta}}
$$

and $\zeta(\eta)$ is the Riemann zeta function. We only need to show that for all $\epsilon>0, \exists M \in \mathbb{N}$ such that for all $N \geqslant M$, we have

$$
\frac{1}{N} U_{w}(N ; d)<-2 \zeta(\eta) \frac{P_{0}^{2}}{d^{\eta}}+\epsilon
$$

First, note that we can write $1 / N U_{w}(N ; d)$ as follows:

$$
\frac{1}{N} U_{w}(N ; d)=-\sum_{i=1}^{N}\left(\frac{P_{0}^{2}}{d^{\eta}} \sum_{\substack{j=1 \\ j \neq i}}^{N} \frac{1}{|i-j|^{\eta}}\right)
$$

Let $K>0$ be such that

$$
\sum_{i=1}^{\infty} \frac{1}{(K+i)^{\eta}}<\epsilon \frac{d^{\eta}}{4 P_{0}^{2}}
$$

For $N>2(K+1)$ we have

$$
\frac{1}{N} U_{w}(N ; d)=-\frac{1}{N} \sum_{i=1}^{N}\left(\frac{P_{0}^{2}}{d^{\eta}} \sum_{\substack{j=1 \\ j \neq i}}^{N} \frac{1}{|i-j|^{\eta}}\right)<-\frac{1}{N} \sum_{i=K+1}^{N-K-1}\left(\frac{P_{0}^{2}}{d^{\eta}} \sum_{\substack{j=1 \\ j \neq i}}^{N} \frac{1}{|i-j|^{\eta}}\right) .
$$

Clearly, for all $i$ such that $K<i<N-K$,

$$
\begin{aligned}
\frac{P_{0}^{2}}{d^{\eta}}(2 \zeta(\eta))-\frac{P_{0}^{2}}{d^{\eta}}\left(\sum_{\substack{j=1 \\
j \neq i}}^{N} \frac{1}{|i-j|^{\eta}}\right) & =\frac{P_{0}^{2}}{d^{\eta}}\left(2 \sum_{j=1}^{\infty} \frac{1}{j^{\eta}}-\sum_{\substack{j=1 \\
j \neq i}}^{N} \frac{1}{|i-j|^{\eta}}\right) \\
& <2 \frac{P_{0}^{2}}{d^{\eta}} \sum_{j=1}^{\infty} \frac{1}{(K+j)^{\eta}}<\frac{\epsilon}{2},
\end{aligned}
$$

where we have used the definition of $K$. Therefore, using Eq. (99) we obtain

$$
\begin{aligned}
\frac{1}{N} U_{w}(N ; d) & <-\frac{1}{N} \frac{P_{0}^{2}}{d^{\eta}} \sum_{i=K+1}^{N-K-1} 2 \zeta(\eta)+\sum_{i=K+1}^{N-K-1} \frac{\epsilon}{2} \\
& =-2 \zeta(\eta) \frac{P_{0}^{2}}{d^{\eta}}+\frac{N-2(K+1)}{N} \frac{\epsilon}{2}+\frac{2(K+1)}{N} 2 \zeta(\eta) \frac{P_{0}^{2}}{d^{\eta}}
\end{aligned}
$$

If we choose $M$ large enough so that $4 \zeta(\eta) \frac{P_{0}^{2}}{d^{\eta}} \frac{K+1}{M}<\epsilon / 2$, for all $N \geqslant M$ we have

$$
\begin{aligned}
\frac{N-2(K+1)}{N} \frac{\epsilon}{2}+4 \zeta(\eta) \frac{P_{0}^{2}}{d^{\eta}} \frac{K+1}{N} & <\epsilon / 2+\epsilon / 2 \\
& =\epsilon
\end{aligned}
$$


which proves the statement of the Lemma for $D=1$.

Lemma A.2: Let $G_{k}(N)$ denote the set of nodes in the frequency band $b_{k}$ corresponding to the optimal frequency band assignment strategy, for an arbitrary spatial configuration of $N$ nodes in $D$ dimensions. Let $N_{k}(N):=\left|G_{k}(N)\right|$ and $\delta:=\min _{i, j} d_{i j}>0$. Then, for each $k=1,2, \cdots, r$, the sequence

$$
\left\{N_{k}(N)\right\}_{N=1}^{\infty}
$$

is unbounded.

Proof: Let $\mathcal{S}(N)=\left\{N_{k}(N) \mid k=1, \cdots, r\right\}$ and $K(N):=\inf \mathcal{S}(N)$. Suppose that the sequence $\{K(N)\}_{N=1}^{\infty}$ is bounded. That is, there exist integers $M, B$ and a specific spatial configuration of the nodes for any $N$, such that $K(N) \leqslant B$ for all $N \geqslant M$. Let $K(N)$ correspond to the frequency band $b_{k^{*}}$ (whereas dependence on $N$ is implicit), i.e., $\left|G_{k}^{*}(N)\right|=K(N)$. Moreover, let $U_{o}^{r}(N)$ denote the optimal network utility corresponding to the $N$ nodes, when there are $r$ frequency bands available to the network. We have

$$
\frac{1}{N} U_{o}^{r}(N)=-\frac{1}{N} \sum_{k \neq k^{*}} \sum_{c_{i}, c_{j} \in G_{k}(N)} \frac{P_{0}^{2}}{d_{i j}^{\eta}}-\frac{1}{N} \sum_{c_{i}, c_{j} \in G_{k^{*}}(N)} \frac{P_{0}^{2}}{d_{i j}^{\eta}}
$$

Suppose that we fix the spatial distribution of the network and the frequency band assignments of all the nodes, but assign arbitrary frequency bands $s_{i}$ to all $c_{i} \in G_{k^{*}}(N)$ such that $s_{i} \neq k^{*}$ for all $i$. Let $U^{r-1}(N)$ denote the network utility corresponding to the foregoing frequency band assignment. We have:

$$
\frac{1}{N} U^{r-1}(N)=-\frac{1}{N} \sum_{k \neq k^{*}} \sum_{c_{i}, c_{j} \in G_{k}(N)} \frac{P_{0}^{2}}{d_{i j}^{\eta}}-\frac{1}{N} \sum_{k \neq k^{*}} \sum_{c_{i} \in G_{k^{*}}(N)} \sum_{c_{j} \in G_{k}(N)} \frac{P_{0}^{2}}{d_{i j}^{\eta}} \delta\left(s_{i}, k\right)
$$

Note that in the foregoing frequency band assignment there are only $r-1$ frequency bands used. Therefore

$$
U^{r-1}(N) \leqslant U_{o}^{r-1}(N)
$$

where $U_{o}^{r-1}(N)$ denotes the optimal network utility corresponding to the $N$ nodes, when there are $r-1$ frequency bands available to the network. We define

$$
g(N):=\frac{1}{K(N)} \sum_{c_{i}, c_{j} \in G_{k^{*}}(N)} \frac{P_{0}^{2}}{d_{i j}^{\eta}}
$$


and

$$
h(N):=\frac{1}{K(N)} \sum_{k \neq k^{*}} \sum_{c_{i} \in G_{k^{*}}(N)} \sum_{c_{j} \in G_{k}(N)} \frac{P_{0}^{2}}{d_{i j}^{\eta}} \delta\left(s_{i}, k\right) .
$$

Hence,

$$
\begin{aligned}
\frac{1}{N} U_{o}^{r}(N) & =\frac{1}{N} U^{r-1}(N)-\frac{K(N)}{N}(g(N)-h(N)) \\
& \leqslant \frac{1}{N} U_{o}^{r-1}(N)-\frac{K(N)}{N}(g(N)-h(N))
\end{aligned}
$$

Clearly, both $g(N)$ and $h(N)$ are upper bounded by $E_{\min }(\eta) P_{0}^{2} / \delta^{\eta}$, where $\delta:=\min _{i, j} d_{i j}>0$ by hypothesis. Hence,

$$
\frac{K(N)}{N}|g(N)-h(N)|<2 E_{\min }(\eta) \frac{P_{0}^{2}}{\delta^{\eta}}\left(\frac{B}{N}\right)
$$

Thus, for $\epsilon>0$ small enough, we can choose $N$ sufficiently large such that

$$
\left|\frac{1}{N} U_{o}^{r}(N)-\frac{1}{N} U_{o}^{r-1}(N)\right| \leqslant \epsilon
$$

Eq. (111) implies that the optimal network utilities of a given network corresponding to $r$ and $r-1$ available frequency bands for a specific spatial configuration may become arbitrarily close.

This is clearly not possible: suppose that there are $r-1$ available frequency bands, $b_{1}, b_{2}, \cdots, b_{r-1}$. Given a fixed spatial configuration, let us consider the optimal frequency band assignment to the nodes $c_{i}, i=1,2, \cdots, N$. Since $\sum_{k} N_{k}(N)=N$, there exists a $k_{0}, 1 \leqslant k_{0} \leqslant r-1$ such that

$$
N_{k_{0}}(N) \geqslant \frac{N}{r-1} \text {. }
$$

Suppose that the nodes in $G_{k_{0}}(N)$ are allowed to choose an additional frequency band, $b_{r}$. Then, according to Theorem 4.2, there exists a frequency band assignment for the nodes in $G_{k_{0}}(N)$, using the two frequency bands $b_{k_{0}}$ and $b_{r}$, for which the network utility of the nodes in $G_{k_{0}}(N)$ is at least half of when all are in frequency band $b_{k_{0}}$. Therefore, this new configuration, with the additional frequency band $b_{r}$, increases the overall network utility by at least

$$
\begin{aligned}
\frac{1}{2}\left(\frac{1}{N} \sum_{c_{i}, c_{j} \in G_{k_{0}}(N)} \frac{P_{0}^{2}}{d_{i j}^{\eta}}\right) & \geqslant \frac{1}{2}\left(\frac{1}{N} \frac{N}{r-1} \frac{1}{(r-1)^{\frac{\eta}{D}}} E_{\min }(\eta) \frac{P_{0}^{2}}{d^{\eta}}\right) \\
& =\frac{1}{2(r-1)^{\frac{\eta}{D}+1}} E_{\min }(\eta) \frac{P_{0}^{2}}{d^{\eta}}
\end{aligned}
$$


for sufficiently large $N$, according to Lemma A.1 and the minimum energy property of of the Bravais lattice $\mathcal{L}^{*}(d)$. Thus

$$
\frac{1}{N} U_{o}^{r}(N) \geqslant \frac{1}{N} U_{o}^{r-1}(N)+\frac{1}{2(r-1)^{\frac{\eta}{D}+1}} E_{\min }(\eta) \frac{P_{0}^{2}}{d^{\eta}}
$$

Eq. (114) implies that $\frac{1}{N} U_{o}^{r-1}(N)$ and $\frac{1}{N} U_{o}^{r}(N)$ can not be arbitrarily close, for any spatial configuration of the nodes, as $N \rightarrow \infty$. This is clearly a contradiction, since Eq. (111) implies that they can be arbitrarily close. Hence, $\{K(N)\}_{N=1}^{\infty}$ is an unbounded sequence. Since $K(N)=$ $\inf \mathcal{S}(N)$ and $\mathcal{S}(N)$ is a finite set of size $r$, we conclude that $\left\{N_{k}(N)\right\}_{N=1}^{\infty}$ is an unbounded sequence for all $k=1,2, \cdots, r$.

\section{APPENDIX B}

\section{DERIVATION OF EQUATION (74)}

Let $\Re:\{1,2, \cdots, r\} \mapsto\{1,2, \cdots, r\}$ be an operator with the following action:

$$
\Re s:=(s+1) \bmod r
$$

for all $s \in\{1,2, \cdots, r\}$. We use the notation $\Re^{k}$ and $\Re^{-k}$ defined by $\Re^{k} s=(s+k) \bmod r$ and $\Re^{-k} s=(r+s-k) \bmod r$, respectively. The Master equation (Eq. (73)) can be therefore expressed as follows:

$$
\begin{aligned}
\frac{\partial}{\partial t} P\left(t ; s_{1}, s_{2}, \cdots, s_{N}\right) & =\sum_{i=1}^{N} \lambda_{i} w_{i}\left(s_{i}\right) \sum_{j=1}^{r-1} P\left(t ; s_{1}, s_{2}, \cdots, \Re^{j} s_{i}, \cdots, s_{N}\right) \\
& -\sum_{i=1}^{N} \sum_{j=1}^{r-1} \lambda_{i} w_{i}\left(\Re^{j} s_{i}\right) P\left(t ; s_{1}, s_{2}, \cdots, s_{N}\right) .
\end{aligned}
$$

for any configuration $\left(s_{1}, s_{2}, \cdots, s_{N}\right)$. Let $f\left(s_{1}, s_{2}, \cdots, s_{N}\right)$ be any scalar function on the statespace. We define the trace operator, $\operatorname{Tr}(\cdot)$, as follows:

$$
\operatorname{Tr}\left(f\left(s_{1}, s_{2}, \cdots, s_{N}\right)\right):=\sum_{\sigma_{1}=1}^{r} \sum_{\sigma_{2}=1}^{r} \cdots \sum_{\sigma_{N}=1}^{r} f\left(\sigma_{1}, \sigma_{2}, \cdots, \sigma_{N}\right)
$$

Hence, the ensemble average of the function $f$ at time $t$ can be written as follows:

$$
\left\langle f\left(s_{1}, s_{2}, \cdots, s_{N}\right)\right\rangle:=\operatorname{Tr}\left(f\left(s_{1}, s_{2}, \cdots, s_{N}\right) P\left(t ; s_{1}, s_{2}, \cdots, s_{N}\right)\right) .
$$


In order to obtain a differential equation for the time evolution of $\left\langle\delta\left(s_{k}, s_{l}\right)\right\rangle$, we multiply both sides of the Eq. (116) by $\delta\left(s_{k}(t), s_{l}(t)\right)$ and take the trace as follows:

$$
\begin{aligned}
& \operatorname{Tr}\left(\frac{\partial}{\partial t}\left(\delta\left(s_{k}, s_{l}\right) P\left(t ; s_{1}, s_{2}, \cdots, s_{N}\right)\right)\right) \\
= & \operatorname{Tr}\left(\sum_{i=1}^{N} \lambda_{i} \delta\left(s_{k}, s_{l}\right) w_{i}\left(s_{i}\right) \sum_{j=1}^{r-1} P\left(t ; s_{1}, s_{2}, \cdots, \Re^{j} s_{i}, \cdots, s_{N}\right)\right) \\
- & \operatorname{Tr}\left(\sum_{i=1}^{N} \sum_{j=1}^{r-1} \lambda_{i} \delta\left(s_{k}, s_{l}\right) w_{i}\left(\Re^{j} s_{i}\right) P\left(t ; s_{1}, s_{2}, \cdots, s_{N}\right)\right) .
\end{aligned}
$$

Note that we have dropped the time dependence of $\delta\left(s_{k}(t), s_{l}(t)\right)$ for notational convenience. The expression on the left hand side can be identified with $\frac{d}{d t}\left\langle\delta\left(s_{k}, s_{l}\right)\right\rangle$. The terms on the right-hand side of Eq. (119) can be written as

$$
\begin{aligned}
& \operatorname{Tr}\left(\sum_{\substack{i=1 \\
i \neq k, l}}^{N} \sum_{j=1}^{r-1} \lambda_{i} \delta\left(s_{k}, s_{l}\right) w_{i}\left(\Re^{-j} s_{i}\right) P\left(t ; s_{1}, s_{2}, \cdots, s_{N}\right)\right) \\
& -\operatorname{Tr}\left(\sum_{\substack{i=1 \\
i \neq k, l}}^{N} \sum_{j=1}^{r-1} \lambda_{i} \delta\left(s_{k}, s_{l}\right) w_{i}\left(\Re^{j} s_{i}\right) P\left(t ; s_{1}, s_{2}, \cdots, s_{N}\right)\right) \\
& +\operatorname{Tr}\left(\sum_{j=1}^{r-1} \lambda_{k} \delta\left(\Re^{-j} s_{k}, s_{l}\right) w_{k}\left(\Re^{-j} s_{k}\right) P\left(t ; s_{1}, s_{2}, \cdots, s_{N}\right)\right) \\
& +\operatorname{Tr}\left(\sum_{j=1}^{r-1} \lambda_{l} \delta\left(s_{k}, \Re^{-j} s_{l}\right) w_{l}\left(\Re^{-j} s_{l}\right) P\left(t ; s_{1}, s_{2}, \cdots, s_{N}\right)\right) \\
& -\operatorname{Tr}\left(\sum_{j=1}^{r-1} \lambda_{k} \delta\left(s_{k}, s_{l}\right) w_{k}\left(\Re^{j} s_{k}\right) P\left(t ; s_{1}, s_{2}, \cdots, s_{N}\right)\right) \\
& -\operatorname{Tr}\left(\sum_{j=1}^{r-1} \lambda_{l} \delta\left(s_{k}, s_{l}\right) w_{l}\left(\Re^{j} s_{l}\right) P\left(t ; s_{1}, s_{2}, \cdots, s_{N}\right)\right)
\end{aligned}
$$

The first two terms clearly cancel each other and the expression reduces to:

$$
\begin{array}{r}
\operatorname{Tr}\left(\sum_{j=1}^{r-1} \lambda_{k} w_{k}\left(\Re^{j} s_{k}\right)\left\{\delta\left(\Re^{j} s_{k}, s_{l}\right)-\delta\left(s_{k}, s_{l}\right)\right\} P\left(t ; s_{1}, s_{2}, \cdots, s_{N}\right)\right) \\
+\operatorname{Tr}\left(\sum_{j=1}^{r-1} \lambda_{l} w_{l}\left(\Re^{j} s_{l}\right)\left\{\delta\left(s_{k}, \Re^{j} s_{l}\right)-\delta\left(s_{k}, s_{l}\right)\right\} P\left(t ; s_{1}, s_{2}, \cdots, s_{N}\right)\right)
\end{array}
$$


It is easy to show that

$$
\sum_{j=1}^{r-1} \lambda_{k} w_{k}\left(\Re^{j} s_{k}\right)\left\{\delta\left(\Re^{j} s_{k}, s_{l}\right)-\delta\left(s_{k}, s_{l}\right)\right\}=\lambda_{k}\left(w_{k}\left(s_{l}\right)-\delta\left(s_{k}, s_{l}\right)\right) .
$$

To see this, note that for $s_{k}=s_{l}$ the above summation becomes

$$
-\sum_{j=1}^{r-1} \lambda_{k} w_{k}\left(\Re^{j} s_{k}\right) \delta\left(s_{k}, s_{l}\right)=\lambda_{k}\left(w_{k}\left(s_{l}\right)-1\right)
$$

and for $\Re^{j} s_{k}=s_{l}$ the summation simply becomes $\lambda_{k} w_{k}\left(\Re^{j} s_{k}\right)=\lambda_{k} w_{k}\left(s_{l}\right)$. Hence, we can combine these two cases as in Eq. (122). Combining the above results, Eq. (119) takes the following form:

$$
\frac{d}{d t}\left\langle\delta\left(s_{k}, s_{l}\right)\right\rangle=-\left(\lambda_{k}+\lambda_{l}\right)\left\langle\delta\left(s_{k}, s_{l}\right)\right\rangle+\left\langle\lambda_{k} w_{k}\left(s_{l}\right)+\lambda_{l} w_{l}\left(s_{k}\right)\right\rangle
$$

If we substitute

$$
w_{k}\left(s_{l}\right)=\frac{\exp \left(-\beta \sum_{j \neq k} J_{k j} \delta\left(s_{l}, s_{j}\right)\right)}{\sum_{m} \exp \left(-\beta \sum_{j \neq k} J_{k j} \delta\left(m, s_{j}\right)\right)}
$$

and

$$
w_{l}\left(s_{k}\right)=\frac{\exp \left(-\beta \sum_{j \neq l} J_{l j} \delta\left(s_{k}, s_{j}\right)\right)}{\sum_{m} \exp \left(-\beta \sum_{j \neq l} J_{l j} \delta\left(m, s_{j}\right)\right)}
$$

into Eq. (124), we get the expression given by Eq. (74), as claimed.

\section{ACKNOWLEDGMENT}

The authors would like to thank Mehrtash Babadi for invaluable comments and discussions, Doina Bein for careful proof-reading and the Associate Editor and anonymous reviewers for their careful and academic evaluation of this work.

\section{REFERENCES}

[1] N. W. Ashcroft and N. D. Mermin, Solid State Physics, Brooks Cole, 1st Edition, Jan. 1976.

[2] B. Babadi, V. Tarokh, "The Impact Of Spectrum Sensing Time On The Performance of the GADIA Algorithm", Proceedings of the 2008 IEEE Symprosia on New Fronties in Dynamic Spectrum Access Networks (DySPAN 2008), Oct. 2008.

[3] B. Babadi, V. Tarokh, "A Distributed Asynchronous Algorithm For Spectrum Sharing In Wireless Ad hoc Networks", Proceedings of the 42nd Annual Conference on Information Sciences and Systems (CISS 2008), Mar. 2008.

[4] B. Babadi, V. Tarokh, "Distributed Dynamic Frequency Allocation In Wireless Networks Under Time-Varying User Activities", Proceedings of the 2008 IEEE Sarnoff Symposium, April, 2008. 
[5] J. Bae, E. Beigman, R. Berry, M. Honig, and R. Vohra, "Sequential Bandwidth and Power Auctions for Distributed Spectrum Sharing," IEEE Journal on Selected Areas in Communication special issue on "Game Theory in Communication Systems," vol. 26, no. 7, pp. 1193-1203, September 2008.

[6] A. V. Bakaev, V. I. Kabanovich, and A. M. Kurbatov, "Antiferromagnetic Potts model Glauber dynamics in the pair approximation", Phys. Lett. A, Vol. 159, 1991.

[7] N. Bambos, "Toward Power-Sensitive Network Architectures inWireless Communications: Concepts, Issues, and Design Aspects”, IEEE Personal Communications, vol. 5, no. 3, p. 50-59, June 1998.

[8] R. J. Baxter, Exactly Solved Models in Statistical Mechanics, Dover Publications, Jan. 2008.

[9] D. Bein, Y. Wen, J. Douglas, S. Phoha, B. B. Madan, and A. Ray, "Distributed Network Control for Mobile Heterogeneous Wireless Sensor Networks", submitted.

[10] P. Bender, P. Black, M. Grob, R. Padovani, N. Sindhushayana, and A. Viterbi, "CDMA/HDR: A Bandwidth-Efficient High-Speed Wireless Data Service for Nomadic Users”, IEEE Communications Magazine, Vol. 38, Issue 7, 2000.

[11] P. K. Biswas and S. Phoha, "Self-Organizing Sensor Networks for Integrated Target Surveillance", IEEE Trans. on Computers, Vol. 55, No. 8, August 2006.

[12] L. Cao and H. Zheng, "Distributed Rule-Regulated Spectrum Sharing", IEEE Journal on Selected Areas in Communications, Special Issue on Cognitive Radio: Theory and Applications, Vol. 26, No. 1, pp 130-145, Jan. 2008.

[13] R. Cendrillon, J. Huang, M. Chiang, and M. Moonen, "Autonomous Spectrum Balancing for Digital Subscriber Lines", IEEE Transactions on Signal Processing, Vol. 55, No. 8, August 2007.

[14] J. H. Conway and N. J. A. Sloane, Sphere Packings, Lattices and Groups, Springer-Verlag NY, First Edition, 1988.

[15] R. Etkin, A. Parekh, and D. Tse, "Spectrum Sharing for Unlicensed Bands", IEEE Journal On Selected Areas In Communications, Vol. 25, No. 3, April 2007.

[16] FCC, FCC 08-260, 14 Nov. 2008, Available at: http://www.fcc.gov/Daily_Releases/Daily_Business/2008/db1117/FCC-08260A1.pdf

[17] W. Feller, Probability Theory, Vol. I, John Wiley, New York, 1957 and Vol. II, 1966.

[18] G. Fettweis, E. Zimmermann, V. Jungnickel, and E. A. Jorswieck, "Challenges in Future Short Range Wireless Systems", IEEE Vehicular Technology Magazine, vol. 1, no. 2, p. 24-31, June 2006.

[19] R. J. Glauber, “Time-dependent statistics of the Ising model”, J. Math. Phys., Vol. 4, pp. 294-307, 1963.

[20] G. H. Hardy, Divergent Series, Providence, American Mathematical Society, 1992.

[21] J. Hicks, A. MacKenzie, J. Neel, J. Reed, “A Game Theory Perspective on Interference Avoidance,” IEEE GlobeCom, Vol. 1, pp. 257-261, Dec. 2004.

[22] J. Huang, R. A. Berry, and M. L. Honig, "Distributed Interference Compensation for Wireless Networks", IEEE Journal On Selected Areas In Communications, Vol. 24, No. 5, May 2006.

[23] J. Huang, R. Berry, and M. Honig, "Auction-based Spectrum Sharing," ACM/Springer Journal of Mobile Networks and Applications (MONET) special issue on WiOpt'04, vol. 11, no. 3, pp. 405-418, June 2006.

[24] K. Huang, Statistical Mechanics, Wiley, 2nd Edition, May 1987.

[25] K. Itô, "Stochastic Integral”, Proc. Imperial Acad. Tokyo, Vol. 20, pp. 519-524, 1994.

[26] N. Karamchandani and M. Franceschetti, "Scaling laws for delay-sensitive traffic in Rayleigh fading networks", Proc. of the Royal Society A 464, pp. 21872205, May 2008.

[27] E. Koutsoupias and C. H. Papadimitriou. "Worst-case equilibria“, In Proceedings of the 16th Annual Symposium on Theoretical Aspects of Computer Science, pp. 404-413, 1999. 
[28] C. Lacatus and D. C. Popescu, "Adaptive Interference Avoidance for Dynamic Wireless Systems: A Game-Theoretic Approach", IEEE Journal on Selected Topics in Signal Processing, vol. 1, no. 1, p. 189-202, June 2007.

[29] J. F. F. Mendes and E. J. S. Lage, "Dynamics of the infinite-ranged Potts model”, Journal of Statistical Physics, Vol. 64, Nos. 3/4, 1991.

[30] K. Leung, B. Kim, "Frequency Assignment for 802.11n Wireless Networks," VTC 2003, vol. 3, pp. 1422-1426, Oct. 6-9 2003.

[31] V. H. MacDonald, “The Cellular Concept”, The Bell Systems Technical Journal, Vol. 58, No. 1, pp. 15-43, Januaray 1979.

[32] I. Oppenheim, K. E. Schuler, and G. H. Weiss, Stochastic Processes in Chemical Physics: The Master Equation, The MIT Press, June 1977.

[33] R. Menon, A. MacKenzie, R. Buehrer, J. Reed, "Game Theory and Interference Avoidance in Decentralized Networks", SDR Forum Technical Conference, Nov. 15-18, 2004.

[34] J. Neel, J. Reed, "Performance of Distributed Dynamic Frequency Selection Schemes for Interference Reducing Networks," Milcom 2006, Washington D.C., Oct 23-25, 2006.

[35] J. Neel, “Synthetic Symmetry In Cognitive Radio Networks," SDR Forum 2007.

[36] J. Neel, "Analysis and Design of Cognitive Radio Networks and Distributed Radio Resource Management Algorithms", PhD Dissertation, Virginia Polytechnic Institute and State University, 2006.

[37] N. Nie, C. Comaniciu, "Adaptive Channel Allocation Spectrum Etiquette for Cognitive Radio Networks," ACM MONET (Mobile Networks and Applications), special issue on "Reconfigurable Radio Technologies in Support of Ubiquitous Seamless Computing", 2006.

[38] C. Peng, H. Zheng, B. Y. Zhao, "Utilization and Fairness in Spectrum Assignment for Opportunistic Spectrum Access", ACM Monet, May 2006.

[39] S. Phoha, G. Mallapragada, Y. Wen, D. Bein, and A. Ray, "Designing a Fusion-Driven Sensor Network to Track Mobile Targets", submitted.

[40] D. C. Popescu and C. Rose, Interference Avoidance Methods for Wireless Systems, Kluwer Academic Publishing, 2004.

[41] D. C. Popescu, O. Popescu, and C. Rose, "Interference Avoidance and Multiaccess Vector Channels", IEEE Transactions on Communications, vol. 55, no. 8, p. 1466-1471, August 2007.

[42] O. Popescu, D. C. Popescu, and C. Rose, "Simultaneous Water Filling in Mutually Interfering Systems", IEEE Transactions on Wireless Communications, vol. 6, no. 3, p. 1102-1113, March 2007.

[43] S. Ramanathan, "A Unified Framework and Algorithm for Channel Assignment in Wireless Networks", Wireless Networks, Volume 5, Issue 2, March 1999.

[44] T. S. Rappaport, Wireless Communications, Prentice Hall, 1st edition, Jan. 1996.

[45] C. Rose, S. Uluks, and R. Yates, "Wireless Systems and Interference Avoidance", IEEE Transactions on Wireless Communications, vol. 1, no. 3, p. 415-428, July 2002.

[46] S. Ross, Stochastic Processes, Wiley, 2nd Edition, 1995.

[47] T. Roughgarden, “Selfish Routing,” PhD Dissertation, Cornell University, May 2002.

[48] M. Steenstrup, “Opportunistic use of radio-frequency spectrum: a network perspective,” DySPAN 2005, Nov. 2005.

[49] R. Steele, L. Hanzo, Mobile Radio Communications, Wiley-IEEE Press, August 1999.

[50] C. W. Sung, K. W. Shum and K.-K. Leung, "Multi-objective power control and signature sequence adaptation for synchronous CDMA systems - a game-theoretic viewpoint", Proceedings of the IEEE International Symposium on Information Theory, July 2003. 
[51] C. W. Sung, K. W. Shum, and K.-K. Leung, "Stability of Distributed Power and Signature Sequence Control for CDMA Systems A Game-Theoretic Framework”, IEEE Trans. on Information Theory, vol. 52, no. 4, p. 1775-1780, April 2006.

[52] A. Sütő, “Crystalline ground states for classical particles”, Physical Review Letters, vol. 95, 265501, 2005.

[53] S. Ulukus and R.D. Yates, "Iterative construction of optimum signature sequence sets in synchronous CDMA systems," IEEE Transactions on Information Theory, vol. 47, no. 5, July 2001, pp. 1989-1998.

[54] W. J. Ventevogel, "On the configuration of a one-dimensional system of interacting particles with minimum potential energy per particle", Physica 92A, pp. 343-361, 1978.

[55] F. Y. Wu, "The Potts model”, Rev. Mod. Phys. 54, 235 - 268, 1982.

[56] Y. Xing, R. Chandramouli, S. Mangold, S. Shankar, "Dynamic spectrum access in open spectrum wireless networks," IEEE Journal on Selected Areas in Communications, vol. 24, No. 3, pp. 626-637, March 2006.

[57] L. Yang, L. Cao, H. Zheng, "Physical Interference Driven Dynamic Spectrum Management," Proceedings of IEEE Symposium on New Frontiers in Dynamic Spectrum Access Networks(DySPAN), October 2008.

[58] W. Yu, G. Ginis, and J. M. Cioffi, “Distributed Multiuser Power Control for Digital Subscriber Lines”, IEEE Journal On Selected Areas In Communications, Vol. 20, No. 5, June 2002.

[59] J. Zhao, H. Zheng, G. Yang, "Distributed Coordination in Dynamic Spectrum Allocation Networks," DySPAN 2005 , pp. 269-278, Nov. 2005.

[60] J. Zhao, H. Zheng and G. Yang, "Spectrum Sharing through Distributed Coordination in Dynamic Spectrum Access Networks', Wireless Communications and Mobile Computing, Special issue on Cognitive and software defined radio, and adaptive wireless communication systems, Vol. 7, Iss. 9, pp. 1061-1075, May 2007.

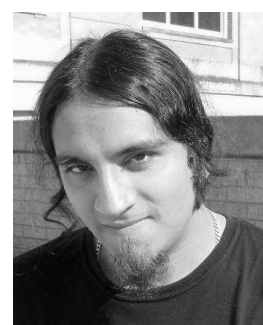

Behtash Babadi (S08) received the BSc. degree in Electrical Engineering from Sharif University of Technology, Tehran, Iran and the MSc. in Engineering Sciences from Harvard University, Cambridge, MA, in 2006 and 2008, respectively.

$\mathrm{He}$ is currently working towards the $\mathrm{PhD}$ degree in Engineering Sciences at the School of Engineering and Applied Sciences, Harvard University, Cambridge, MA. His research interests include dynamic spectrum access networks, adaptive signal processing and compressed sensing.

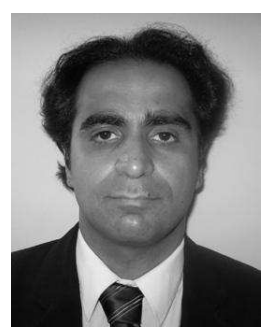

Vahid Tarokh (M97-SM02-F09) worked at AT\&T Labs-Research until August 2000, where he was the head of the Department of Wireless Communications and Signal Processing. He then joined the Department of Electrical Engineering and Computer Sciences (EECS) at MIT as an associate professor. In 2002, he joined Harvard University as a professor and senior fellow.

Dr. Tarokh's research interest is mainly focused in the areas of information theory, signal processing, communications and networking. He has received a number of awards, and holds 2 honorary degrees. 